\title{
ADMM-based Market Clearing and Optimal Flexibility Bidding of Distribution-level Flexibility Market for Day-Ahead Congestion Management of Distribution Networks
}

Shen, Feifan; Wu, Qiuwei; Jin, Xiaolong; Zhou, Bin; Li , Canbing; Xu, Yan

Published in:

IEEE Transactions on Industrial Informatics

Link to article, DOI:

10.1016/j.ijepes.2020.106266

Publication date:

2020

Document Version

Peer reviewed version

Link back to DTU Orbit

Citation (APA):

Shen, F., Wu, Q., Jin, X., Zhou, B., Li , C., \& Xu, Y. (2020). ADMM-based Market Clearing and Optimal Flexibility Bidding of Distribution-level Flexibility Market for Day-Ahead Congestion Management of Distribution Networks. IEEE Transactions on Industrial Informatics, 123, [106266]. https://doi.org/10.1016/j.ijepes.2020.106266

\section{General rights}

Copyright and moral rights for the publications made accessible in the public portal are retained by the authors and/or other copyright owners and it is a condition of accessing publications that users recognise and abide by the legal requirements associated with these rights.

- Users may download and print one copy of any publication from the public portal for the purpose of private study or research.

- You may not further distribute the material or use it for any profit-making activity or commercial gain

- You may freely distribute the URL identifying the publication in the public portal 


\title{
ADMM-based Market Clearing and Optimal Flexibility Bidding of Distribution-level Flexibility Market for Day-Ahead Congestion Management of Distribution Networks
}

Feifan Shen ${ }^{\mathrm{a}, \mathrm{c}}$, Qiuwei $\mathrm{Wu}^{\mathrm{a}}$, Xiaolong Jin ${ }^{\mathrm{a}}$, Bin Zhou ${ }^{\mathrm{b}}$, Canbing $\mathrm{Li}^{\mathrm{b}}$, Yan $\mathrm{Xu}^{\mathrm{c}}$

${ }^{a}$ Center for Electrical Power and Energy (CEE), Department of Electrical Engineering, Technical University of Denmark, Kgs. Lyngby, 2800, Denmark

${ }^{b}$ College of Electrical and Information Engineering, Hunan University, Changsha 410082, China

${ }^{c}$ School of Electrical and Electronic Engineering, Nanyang Technological University, 50 Nanyang Avenue, Singapore, 639798

*Corresponding author: email: qw@elektro.dtu.dk

\begin{abstract}
Nowadays, the massive deployment of distributed energy resources in distribution networks poses more operational challenges such as network congestion to distribution system operators. To cope with these challenges in an economically efficient way, the distribution system operator can utilize demand-side flexibility traded in the local flexibility market to manage the distribution network. In the existing distribution-level flexibility market frameworks, the market operator is assumed to have access to network parameters in order to ensure the market clearing solution being technically feasible from the operational point of view. To protect the privacy of network parameters, this paper proposes an alternating direction method of multipliers -based market clearing strategy, in which the market operator communicates with the distribution system operator to clear the market such that the market clearing solution respects network operation constraints without revealing network parameters to the market operator. In addition, in the existing flexibility bid formulations, the energy payback condition is determined without considering operation constraints of flexibility resources and the flexibility cost has not been considered. To fill this gap, this paper formulates an optimal flexibility bidding model for aggregators, which carefully models the energy payback condition and enables the aggregator to receive the maximum revenue with flexibility costs considered. The case studies were conducted on the Roy Billinton Test System with electrical vehicles and heat pumps as flexibility resources. The results demonstrate that the proposed distribution-level flexibility market framework is effective to perform day-ahead congestion management of distribution networks and is profitable to aggregators and end-users.
\end{abstract}

Key Words: Alternating direction method of multipliers (ADMM), congestion management, distribution network, flexibility service bid, local flexibility market.

\section{Nomenclature}

Set:

$$
\begin{aligned}
& T, T^{\mathrm{c}}, T^{\mathrm{rb}} \begin{array}{l}
\text { Sets of the day-ahead scheduling hours, } \\
\text { congestion hours, and allowable payback hours }
\end{array} \\
& N^{\mathrm{nd}}, N^{l} \quad \text { Sets of nodes and transmission lines } \\
& N^{\mathrm{ev}}, N^{\mathrm{hp}} \quad \text { Sets of EVs and HPs } \\
& N^{\mathrm{ag}} \quad \text { Set of aggregators } \\
& N_{a, n}^{\mathrm{ev}}, N_{a, n}^{\mathrm{hp}} \text { Sets of EVs and HPs belonging to } a \text {-th }\left(a \in N^{\mathrm{ag}}\right) \\
& \quad \text { aggregator at node } n \in N^{\mathrm{nd}}
\end{aligned}
$$

\section{Variables:}

$f_{t, a, n}^{\mathrm{fle}} \quad$ The amount of available flexibility at hour $t$ stipulated in the bid provided by $a$-th aggregator at node $n$ aggregator at node $n$ at hour $t$

$r_{t, a, n}^{\mathrm{rb}} \quad$ The amount of payback power required by $a$-th aggregator at node $n$ at hour $t$

$f_{t, e}^{\mathrm{ev}}, f_{t, h}^{\mathrm{hp}}$ The amount of flexibility provided by $e$-th EV and $h$-th HP at hour $t$, respectively

$r_{t, e}^{\mathrm{ev}}, r_{t, h}^{\mathrm{hp}} \quad$ The amount of payback power required by $e$-th EV and $h$-th HP at hour $t$, respectively

$p_{t, e}^{\mathrm{ev}}, p_{t, h}^{\mathrm{hp}} \quad$ Charging power of $e$-th EV and power 
consumption of $h$-th HP at hour $t$, respectively

$K_{t, h}^{\mathrm{h}}, K_{t, h}^{\mathrm{u}}$ Household inside and outside temperature of the house with $h$-th HP at hour $t$, respectively

$x_{t, a, n}^{\mathrm{rb}} \quad$ The binary variable representing the status of payback power, the $a$-th aggregator at node $n$ can have payback power at hour $t$ if $x_{t, a, n}^{\mathrm{rb}}=1$

$x_{t, a, n}^{\mathrm{fle}} \quad$ The procurement percentage of the flexibility service bid provided by $a$-th aggregator at node $n$ at hour $t, x_{t, a, n}^{\mathrm{fle}} \in[0,1]$

$F_{t, l} \quad$ Active power flow of line $l \in N^{l}$ at hour $t$

$p_{t, n}, q_{t, n} \quad$ Actual active and reactive power consumption at node $n$ at hour $t$, respectively

$f_{t, a, n}^{\mathrm{ag}} \quad$ The amount of flexibility purchased from $a$-th aggregator and node $n$ at hour $t$

$\lambda_{e}^{\mathrm{ev}}, \lambda_{h}^{\mathrm{hp}} \quad$ Increased day-ahead energy costs of $e$-th EV and $h$-th HP, respectively

Parameters:

$e_{e}^{\min / \max } \quad$ Lower and upper limits of the SOC level of $e$-th EV

$e_{e, t_{0}} \quad$ Initial SOC level of $e$-th EV

$p_{e}^{\mathrm{ev}, \min / \max }$ Lower and upper limits of $e$-th EV charging power

$d_{t, e} \quad$ Driving distance of $e$-th EV at hour $t$

$s_{t, e} \quad$ The binary value representing the charging availability of $e$-th at hour $t$

$c_{h}^{\text {cop }} \quad$ The performance coefficient of $h$-th HP

$k_{1}, k_{2} \quad$ The heat transfer coefficients

$p_{h}^{\text {hp, } \min / \max }$ Lower and upper limits of $h$-th HP power consumption

$K_{h}^{\mathrm{h}, \min / \max }$ Lower and upper limits of the household inside temperature of the house with $h$-th HP

$\lambda_{a, n}^{\text {fle }} \quad$ The bidding price of the flexibility service bid provided by $a$-th aggregator at node $n$

$c_{t, e}^{\mathrm{ev}}, c_{t, h}^{\mathrm{hp}} \quad$ Cost coefficients of $e$-th EV and $h$-th HP at hour $t$, respectively

$\hat{p}_{t, e}^{\mathrm{ev}}, \hat{p}_{t, h}^{\mathrm{hp}} \quad$ Baseline charging power and power consumption of $e$-th EV and $h$-th HP at hour $t$, respectively

$\hat{p}_{t . n} \quad$ Baseline power consumption at node $n$ at hour $t$

$\alpha \quad$ The upper limit of the payback percentage

$\lambda_{t}^{\mathrm{DA}} \quad$ The forecasted day-ahead energy prices at hour $t$

$G_{n, l} \quad$ The element at $n$-row and $l$-th column of the incidence matrix

$\cos \left(\phi_{n}\right)$ The power factor at node $n$

$F_{l}^{\max } \quad$ Capacity of line $l$

$V^{\mathrm{s}}, V^{\mathrm{min}}$ The voltage magnitude at the substation and the lower limit of the voltage magnitude

$z_{i, j} \quad$ The impedance of the transmission line between nodes $i$ and $j$

$f_{t, a, n}^{\mathrm{pr}} \quad$ The amount of flexibility purchased from $a$-th aggregator and node $n$ at hour $t$ after the DFM clearing

$\rho \quad$ Penalty parameter of the ADMM algorithm

$\tau, \zeta \quad$ Update parameters

Acronyms:

DER Distributed energy resources

EV Electrical vehicle

HP Heat pump

DSO Distribution system operator

DFM Distribution-level flexibility market

DT Dynamic tariff

DS Dynamic subsidy

RT Real-time

ESS Energy storage system

DG Distributed generation

BRP Balance responsible party

NLP Nonlinear programming

MIQP Mixed integer quadratic programming

MILP Mixed integer linear programming

ADMM Alternating direction method of multipliers

OPF Optimal power flow

$\mathrm{AC} / \mathrm{DC}$ Alternating current/direct current

FRT Flexibility requirement table

LP Load point 


\section{Introduction}

In order to achieve the Danish government's ambitious vision of realizing independence from fossil fuels by 2050, i.e., “Energy strategy 2050” [1], distributed energy resources (DERs) such as electrical vehicles (EVs) and heat pumps (HPs) have been massively deployed in distribution networks. This leads to a significant paradigm shift of the network and poses more operational challenges to the distribution system operators (DSOs) [2]. One major concern of the DSO is the network congestion caused by the uncoordinated power consumption of DERs, e.g., simultaneous charging of EVs. In addition to network reinforcement, the DSOs can use market-based demand response programs to utilize demand-side flexibility for resolving day-ahead congestion in an economically efficient way [3]. The demand response program is established to change electricity consumption of end-users in response to changes of electricity prices over time or given incentives [4]. The marketbased demand response programs can be roughly categorized into two types [5]: 1) price-based programs; and 2) incentivebased programs.

\subsection{Literature review}

\subsubsection{Price-based programs}

In price-based programs, end-users response to changes of electricity prices by modifying power consumption patterns. In the dynamic tariff (DT)-based methods in [6]-[10], the DSO publishes time-dependent DTs at different locations of the network, which leads to higher final electricity prices (base price plus DTs) at peak hours. As a result, the aggregators or endusers shift power consumption from peak hours to off-peak hours to minimize energy costs, which consequently helps mitigate congestion. In [6], a DT-based framework was developed to coordinate EV charging to alleviate congestion. However, due to the linear formulation of the DT optimization model, the model might have multiple optimal solutions in the case of with same energy prices in different hours, which will cause inconsistency between the solution at the DSO side and solutions at the aggregator side and consequently lead to a failure of congestion management. In [7], a quadratic programming (QP) formulation of the DT concept was developed to resolve the multiple solution issue. The proposed DT frameworks in [6], [7] rely on the DSO to accurately predict uncertain flexible demands. This might not work in some cases due to the prediction errors. In [8], an uncertainty management method based on the DT framework was developed to handle uncertainties of flexible demands. A sensitivity-based iterative procedure was used to quantify the probability distribution of the forecast error in order to reserve a line capacity margin to deal with uncertainties. In [9], a robust DT model was developed to account for uncertainties of flexible demands. To resolve the solution conservatism obtained with the robust DT model, a sensitivity-based real-time (RT) adjustment method was used in a receding horizon to adjust the obtained DT in RT. In order to relieve the DSO from predicting information from the demand side, distributed optimizations for the DT framework were proposed in [9] and [10], in which the DTs are obtained through an iterative procedure between the DSO and aggregators such that the DSO does not need to predict information at the demand side.

In [11], a line shadow price method was proposed, which works similarly to the DT method with the shadow price obtained through an interactive process between the DSO and aggregators or end-users. In the DT and shadow price methods, tariffs are published in peak hours to increase final prices. In contrast, a dynamic subsidy (DS) method was proposed in [12], in which the subsidies are given to reduce final prices (base price minus subsidies) in the off-peak hours. The DS method has a similar congestion management framework as the DT method and uses a different model to calculate DSs.

\subsubsection{Incentive-based programs}

In the incentive-based programs, end-users are rewarded by modifying power consumption profiles through the distribution-level flexibility market (DFM). A bilateral-contract-based DFM was proposed in [13], in which trade processes are carried out between the DSO and aggregators and between the aggregator and end-users. This market is based on a network of bilateral flexibility contracts with multiple contracts between each pair of agents that are able to trade with one other. In [14]-[20], the pool-based DFM framework was studied, where flexibility sellers and buyers offer bids in the market and the 
market operator clears the market for different operational purposes. The proposed DFM frameworks in [14]-[20] share a similar operational mechanism that the aggregators or end-users bid flexibility service in the DFM and then the market operator clears the market to satisfy the DSO's flexibility requirements with different market clearing models. A DFM framework for day-ahead and intraday congestion management was proposed in [15], in which market participants consist of the DSO, market operator, balance responsible party (BRP), and aggregator. The DSO is the only flexibility buyer in the day-ahead DFM for congestion management, whereas the BRP participates in the intraday DFM and competes with the DSO for flexibility to minimize imbalance costs. The day-ahead and intraday market clearing problems formulated as mixed integer linear programming (MILP) models are solved by the market operator with the social welfare maximization. This DFM framework is complemented with a real-time dispatching strategy in [16]. If the market-based solution fails to resolve congestion, the DSO takes over the control of flexible resources to adjust energy schedules. A similar DFM framework was proposed in [17] to resolve day-ahead congestion with micro-grids being flexibility service providers and the DSO playing the role of the market operator. The micro-grid agent aggregates small size distributed generations (DGs), energy storage systems (ESSs) and customers to provide flexibility service. The market clearing problem formulated as a non-linear programming (NLP) model is solved by the DSO with the minimum flexibility procurement cost.

The above-mentioned DFM frameworks for day-ahead congestion management are executed before the operation of the day-ahead energy market. As such, the DFM clearing solutions are used to adjust original energy schedules so that the resulting schedules after the day-ahead energy market clearing lead to no congestion. In contrast, the DFM frameworks proposed in [18]-[20] operate after the day-ahead market clearing if the accepted energy schedules result in congestion. The market clearing problems in [18]-[20] formulated as NLP models are solved by the DSO to minimize flexibility procurement costs. Moreover, a real-time DFM and the probability analysis-based method were developed in [19] and [20] to deal with uncertainties of flexibility resources. However, in these DFM frameworks, the imbalance cost due to rescheduling energy schedules after the day-ahead energy market clearing is not considered.

\subsection{Motivations}

In the DT and shadow price methods [6]-[11], end-users, who help mitigate congestion, need to pay congestion costs instead of getting rewards. In addition, in these price-based methods [6]-[12], the end-users receive different tariffs or subsidies because of their locations in the network, which is against the non-discrimination rule [12]. The DFM-based method can resolve the above-mentioned issues. In the DFM framework, end-users helping mitigate congestion receive rewards that are determined by the flexibility service provided. However, there are two issues when applying the existing DFM frameworks to resolve day-ahead congestion.

The first one is privacy information protection. In the existing DFM frameworks, it is assumed that the market operator has access to network parameters in order to ensure the market clearing solution being technically feasible, i.e., the market clearing solution satisfies network operation constraints, which may compromise the privacy protection [17]-[20]. The second issue is the optimal formulation of the flexibility service bid. One key element of the flexibility service bid is the energy payback condition, i.e., the power payback amount and hour [21]. However, these conditions are determined without modeling operation constraints of flexibility resources in the existing DFM frameworks [17]-[20]. Moreover, the flexibility cost namely the payment to flexibility resources for providing flexibility has not been considered in the bid formulation, which may make the end-users or aggregators not be willing to participate in the DFM because their revenues are uncertain.

This paper aims to improve the current DFM framework. To improve privacy information protection, an alternating direction method of multipliers (ADMM)-based market clearing strategy is proposed, in which the market operator communicates with the DSO to clear the DFM so that the market clearing solution respects network operational constraints without revealing network parameters to the market operator. The ADMM as a distributed algorithm has been widely used to solve convex problems in power system applications [22]-[27], such as load restoration [23], convexified optimal power flow 
[24], [25], voltage control [26], and demand response [27]. In [23], a distributed load restoration strategy was proposed using the ADMM algorithm. In the proposed strategy, the load restoration problem was decomposed into sub-problems for each node and solved by agents at each node through exchanging information between neighbor agents. In [24], the convexified alternating current $(\mathrm{AC})$ optimal power flow (OPF) problem was decomposed and solved by the ADMM algorithm in a distributed manner. In the algorithm, the closed-form solutions of subproblems at each node are obtained, which significantly improve the computation efficiency. The direct current (DC)-OPF problem was solved by the consensus ADMM in [25]. Based on the consensus ADMM framework, three distributed algorithms, i.e., distributed DC-OPF with a central controller, fully decentralized DC-OPF, and distributed DC-OPF with the accelerated ADMM, were developed and compared with respect to convergence performance and communication architecture. In [26], a distributed reactive power control scheme based on the consensus ADMM was designed for voltage control of the wind farm cluster. Using the proposed distributed control scheme, the fair reactive power sharing among wind farms can be achieved to regulate voltages. Moreover, the computation burden of wind farm controllers and communication costs are reduced. In [27], an ADMM-based distributed residential load control strategy was proposed for peak load shaving considering operation constraints of unbalanced distribution networks and the privacy information protection. In addition, this paper proposes an optimal bidding strategy for the aggregator under a given bidding price. The optimal bidding problem is formulated as a mixed integer linear programming model (MILP) that determines the energy payback condition considering operation constraints of flexibility resources and maximizes the aggregator's revenues with flexibility costs included. If the flexibility bid is accepted in the DFM, the aggregator and endusers can receive revenues accordingly.

In summary, compared with the existing DT and shadow price methods [6]-[11], the end-users helping mitigate congestion receive rewards instead of paying congestion costs in the proposed DFM framework. Compared with the DS method [12], the proposed DFM framework has no locational discrimination issue because the end-users located in a network receive the same system energy price. Compared with the existing DFM frameworks [14]-[20], the proposed framework has two improvements: 1) the privacy information protection is improved by introducing the ADMM-based market clearing strategy, in which the system parameters do not need to be revealed to the market operator; and 2) the energy payback conditions of flexibility resources and flexibility costs are considered and carefully modeled in the proposed optimal flexibility bidding strategy.

The contributions of this paper are summarized as follows.

1) Propose an optimal flexibility bidding strategy for aggregators in the DFM, which carefully models energy payback conditions of flexibility resources and enables the aggregator to receive the maximum revenue with the flexibility cost included;

2) Develop an ADMM-based market clearing strategy for the DFM, in which the market clearing solution respects network operational constraints without revealing network parameters to the market operator.

The rest of this paper is organized as follows. Section 2 presents the concept of the DFM and the proposed operational mechanism of day-ahead congestion management with the DFM. Section 3 presents three model formulations for the baseline energy schedule formulation, optimal flexibility bidding, and market clearing problem. Section 4 presents the proposed ADMM-based market clearing strategy. Case study results are discussed in Section 5, followed by conclusions in Section 6.

\section{Distribution-level Flexibility Market}

\subsection{Concept of the DFM}

The DFM is a flexibility trading platform where different parties trade flexibility in a geographically limited area, such as a community or a city [28]. A schematic overview of a DFM is shown in Fig. 1, where DFM participants are the aggregator, DSO, and market operator. The roles and responsibilities of the participants are described as follows. 


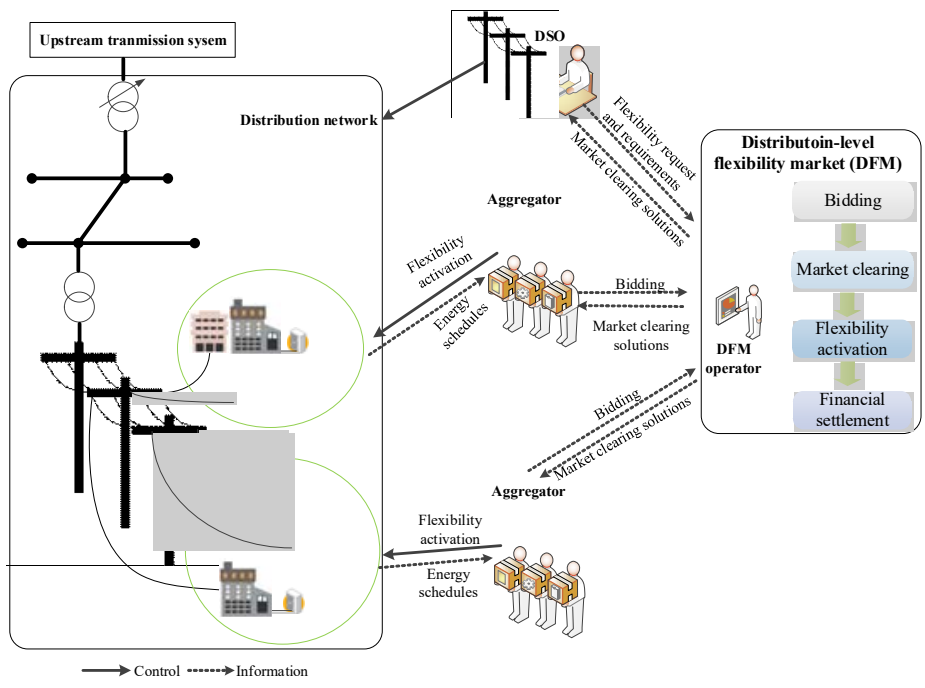

Fig. 1 Schematic overview of a distribution-level flexibility market

1) DSO: The DSO is responsible for the secure operation of the distribution network and effective service delivery to customers. The DSO can procure flexibility in the DFM for different operational purposes, such as congestion management and line loss reduction.

2) Aggregator: The aggregator acts as an intermediary between customers and other DFM participants. Since the individual customer has limited negotiation power in the DFM due to its small volume of flexibility, it is necessary to have an aggregator that can gather flexibility from customers, formulate flexibility service bids, and trade flexibility in the DLM. The aggregator and customers reach an agreement that the aggregator can schedule flexibility sources of customers.

3) Market operator: The DFM operator is an independent entity running the DFM. The DFM operator is responsible for the market clearing process that determines trading results, i.e., the procurement cost and amount of flexibility traded.

The flexibility trading process is also shown in Fig. 1. Firstly, after the DSO sends a flexibility request and publishes flexibility requirements, the aggregators formulate flexibility service bids and offer them in the DFM. Secondly, the market operator clears the DFM to determine accepted flexibility service bids to meet the DSO's flexibility requirements. Thirdly, according to the market clearing solution, the aggregators schedule flexibility resources to provide committed flexibility. Finally, flexibility transactions are completed through financial settlement. The DSO pays flexibility procurement costs while the aggregators and customers receive revenues for providing flexibility service.

\subsection{The mechanism of day-ahead congestion management with the DFM}

This study focuses on the application of the DFM to day-ahead congestion management in distribution networks. Congestion management in distribution networks has two categories [29]: 1) overload management; and 2) voltage management. The proposed operational mechanism of day-ahead congestion management with the DFM is shown in Fig. 2 and described as follows.

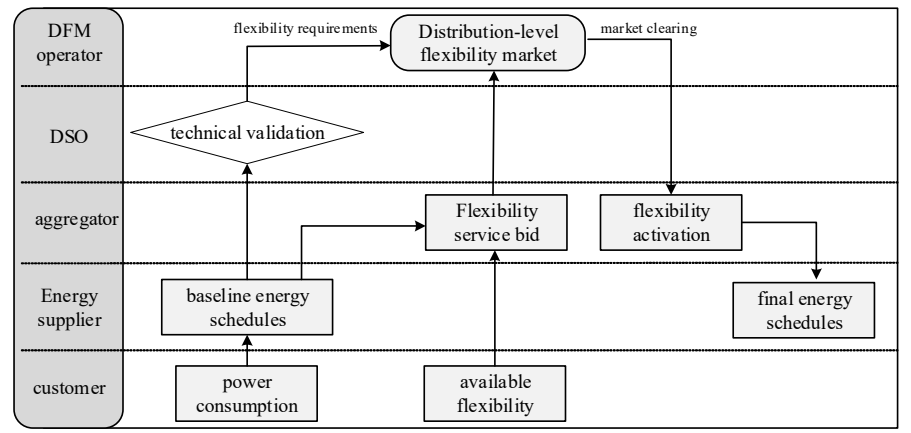

Fig. 2 The proposed operational mechanism of day-ahead congestion management with the DLM 
Firstly, based on the forecasted day-ahead spot prices, energy suppliers make baseline energy schedules for customers with the minimum energy costs. The role of the energy supplier is to purchase energy in the day-ahead energy market on behalf of customers. It is assumed that the energy supplier and aggregator are two different entities; otherwise, the aggregator could intentionally make baseline energy schedules that result in congestion and then make profits by selling flexibility in the FLM to deal with congestion that is originally caused by the aggregator itself.

Secondly, the baseline energy schedules are sent to the DSO for technical validation. The DSO conducts the power flow analysis to examine if the schedules are technically feasible. Once the DSO identifies there is congestion in the following day of operation, the DSO sends a flexibility request to the DFM and publishes a flexibility requirement table (FRT). Table 1 is an example of the FRT, which stipulates congestion hours $\left(t_{6}, t_{18}\right)$, load points (LPs) requested to provide flexibility $\left(\mathrm{LP}_{4-5}\right)$, the minimum amount of flexibility required from $\mathrm{LP}_{4-5}$ at each congestion hour (100 $\mathrm{kW}$ at $t_{6}, 120 \mathrm{~kW}$ at $\left.t_{18}\right)$, allowable energy payback hours $\left(t_{21}-t_{24}\right)$, the upper limit of the energy payback percentage $(150 \%)$ that is equal to the amount of payback power divided by the amount of flexibility provided, and the maximum bidding price $(1.5 \mathrm{DKK} / \mathrm{kW})$ at which the DSO is willing to pay for flexibility. The determination of the key parameters in the FRT is described below,

- Congestion hours and load points requested to provide flexibility

After receiving the baseline energy schedules, the DSO can identify congestion hours and lines using the power flow analysis. According to the topology information, the DSO can determine those load points that are able to provide flexibility to resolve congestion.

- The minimum and maximum amount of flexibility required

The minimum and maximum amount of flexibility required are approximate values. The two values provide a reference for aggregators to provide the appropriate amount of flexibility. The actual amount of flexibility required for resolving congestion is obtained after the DFM clearing. For overload management, the amount of flexibility required for resolving overloading issue is equal to the line overload amount. For voltage management, the amount of flexibility required at node $j$ for resolving the over/under-voltage issue at node $i$ can be calculated using the nodal voltage deviation from voltage limits at node $i$ and the voltage sensitivity of node $i$ with respect to the power injection at the node $j$. Therefore, the minimum and maximum amount of flexibility required for voltage management can be obtained according to the amount of flexibility required at each node. The calculation of the voltage sensitivity will be detailed in Section 5 .

- The upper limit of the energy payback percentage and maximum bidding price

According to historical data of flexibility service bids, the DSO can stipulate the upper limits of the payback percentage and bidding price for the flexibility service bid.

- Allowable energy payback hours

With the approximated maximum amount of flexibility required and the stipulated upper limit of the payback percentage, the approximated maximum amount of payback power can be calculated, based on which the DSO conducts the power flow analysis to select the allowable payback hours. In these payback hours, energy payback can occur without causing new congestion. To deal with approximation errors when selecting payback hours, a security margin can be reserved in the power flow analysis.

Table 1. Flexibility requirement table

\begin{tabular}{c|c|c|c|c|c}
\hline \hline \multirow{2}{*}{ load points } & \multicolumn{2}{|c|}{$\begin{array}{c}\text { the minimum amount of flexibility } \\
\text { required at congestion hours }[\mathrm{kW}]\end{array}$} & allowable payback hours & $\begin{array}{c}\text { upper limit of } \\
\text { payback percentage }\end{array}$ & $\begin{array}{c}\text { maximum bidding price } \\
{[\mathrm{DKK} / \mathrm{kW}]}\end{array}$ \\
\hline \multirow{2}{*}{$\mathrm{LP}_{4-5}$} & $t_{6}$ & $t_{18}$ & $t_{21}-t_{24}$ & $150 \%$ & $1.5 \mathrm{DKK}$ \\
\cline { 2 - 3 } & 100 & 120 & & \\
\hline \hline
\end{tabular}

According to the FRT, the aggregators formulate flexibility service bids considering operation constraints of flexibility 
sources, e.g., EVs and HPs, and submit the bids in the DFM. Table 2 lists two examples of flexibility service bids provided by two aggregators $\left(a g_{1}, a g_{2}\right)$ at $\mathrm{LP}_{4}$. The flexibility service bid stipulates the bidding price, amount and locations of flexibility provided at each congestion hour, energy payback hour and amount of payback power. For simplification of the bid formulation, the aggregator is assumed to have payback power at one hour only. After the flexibility bidding process, the market operator clears the DFM to determine accepted bids. With the trading result and baseline energy schedules, the aggregators reschedule flexibility sources to provide committed flexibility. Then, the final energy schedules will be submitted to the day-ahead energy market. Finally, financial settlement regarding flexibility trading is carried out based on the pay-asbid rule. The DSO pays flexibility procurement costs and the aggregators receive payments according to bidding prices. Moreover, the customers providing flexibility service receive payments from the aggregators accordingly.

Table 2. Flexibility service bids

\begin{tabular}{c|c|c|c|c|c}
\hline \hline \multirow{2}{*}{ aggregator } & \multirow{2}{*}{ price $[\mathrm{DKK} / \mathrm{kW}]$} & \multirow{2}{*}{ load points } & \multicolumn{2}{|c|}{ the amount of flexibility [kW] } & \multicolumn{2}{|c}{ payback hour and power [kW] } \\
\cline { 4 - 6 } & & & $t_{6}$ & $t_{18}$ & $t_{21}$ \\
\hline$a g_{1}$ & 0.55 & $\mathrm{LP}_{4}$ & 20 & 30 & 50 \\
$a g_{2}$ & 0.65 & $\mathrm{LP}_{4}$ & 30 & 40 & 55 \\
\hline \hline
\end{tabular}

\section{Model Formulations}

This section presents four optimization models. The first optimization model is to obtain the baseline day-ahead energy schedules for customers with minimal costs according to the forecasted day-head energy prices. The second optimization model is for the aggregator to formulate the optimal flexibility service bid considering operation constraints of flexible resources. The third optimization model is the DFM clearing model that determines acceptable flexibility service bids to satisfy the DSO's flexibility requirements. The fourth optimization of for the aggregator to reschedule its contractual customers based on the trading results in the DFM.

\subsection{Baseline day-ahead energy schedules}

Due to the deployment of EVs and HPs at the residential buildings [30], [31] and the large potential to provide demand flexibility [32], [33], EVs and HPs are considered as flexibility providers in this study. Suppose that each customer owns an EV and a HP. The baseline energy schedules of EVs and HPs are obtained by minimizing day-ahead energy costs while satisfying operational constraints. The optimization model is formulated as below,

$$
\begin{aligned}
& \text { Min } \sum_{t \in T} \lambda_{t}^{\mathrm{DA}}\left(\sum_{e \in N^{\mathrm{ev}}} p_{t, e}^{\mathrm{ev}}+\sum_{h \in N^{\mathrm{hp}}} p_{t, h}^{\mathrm{hp}}\right) \\
& e_{e}^{\mathrm{min}} \leq \sum_{t \leq t}\left(p_{t, e}^{\mathrm{ev}}-d_{t, e}\right)+e_{e, t_{0}} \leq e_{e}^{\mathrm{max}} ; \forall t \in T, e \in N^{\mathrm{ev}} \\
& s_{t, e} p_{e}^{\mathrm{ev}, \min } \leq p_{t, e}^{\mathrm{ev}} \leq s_{t, e} p_{e}^{\mathrm{ev}, \max } ; \forall t \in T, e \in N^{\mathrm{ev}} \\
& \sum_{t \in T} p_{t, e}^{\mathrm{ev}}=\sum_{t \in T} d_{t, e} ; \forall e \in N^{\mathrm{ev}} \\
& c_{h}^{\mathrm{cop}} p_{t, h}^{\mathrm{hp}}-k_{1}\left(K_{t, h}^{\mathrm{h}}-K_{t, h}^{\mathrm{u}}\right)=k_{2}\left(K_{t, h}^{\mathrm{h}}-K_{t-1, h}^{\mathrm{h}}\right) ; \forall t \in T, h \in N^{\mathrm{hp}} \\
& K_{h}^{\mathrm{h}, \min } \leq K_{t, h}^{\mathrm{h}} \leq K_{h}^{\mathrm{h}, \max } ; \forall t \in T, h \in N^{\mathrm{hp}} \\
& p_{h}^{\mathrm{hp}, \min } \leq p_{t, h}^{\mathrm{hp}} \leq p_{h}^{\mathrm{h}, \max } ; \forall t \in T, h \in N^{\mathrm{hp}}
\end{aligned}
$$

The objective function (1) is to minimize day-ahead energy costs of EVs and HPs based on the forecasted day-ahead spot prices $\lambda_{t}^{\mathrm{DA}}$, where $p_{t, e}^{\mathrm{ev}}$ and $p_{t, h}^{\mathrm{hp}}$ are $e$-th EV and $h$-th HP power consumption at hour $t$, respectively. Constraint (2) limits the state of charge (SOC) level of the EV, where $e_{e}^{\min }$ and $e_{e}^{\max }$ are the lower and upper bounds of $e$-th EV's SOC level, $e_{e, t_{0}}$ is the $e$-th EV's initial SOC level, and $d_{t, e}$ is the power consumption of $e$-th EV at hour $t$. Constraint (3) limits the EV charging 
power considering the EV charging availability, where $p_{e}^{\mathrm{ev}, \min }$ and $p_{e}^{\mathrm{ev}, \max }$ are the lower and upper bounds of $e$-th EV charging power, and $s_{t, e}$ is the charging availability of $e$-th EV at hour $t$. Constraint (4) represents that the total charging power is equal to the total power consumption during the optimization horizon. Constraint (5) represents the household thermal balance equation, where $c_{h}^{\text {cop }}$ is the performance coefficient of $h$-th HP, $k_{1}$ and $k_{2}$ are heat transfer coefficients, and $K_{t, h}^{\mathrm{h}}$ and $K_{t, h}^{\mathrm{u}}$ are household inside and outside temperature of the house with $h$-th HP at hour $t$, respectively. Constraint (6) limits the household inside temperature within a thermal comfort range, where $K_{h}^{\mathrm{h}, \min }$ and $K_{h}^{\mathrm{h} \text {,max }}$ are the lower and upper bounds of household inside temperature of the house with $h$-th HP. Constraint (7) limits the HP power consumption, where $p_{h}^{\text {hp,min }}$ and $p_{h}^{\text {hp,max }}$ are lower and upper bounds of HP power consumption, respectively.

\subsection{Optimal flexibility bidding}

Based on the baseline energy schedules and the FRT, the aggregators formulate flexibility service bids by rescheduling EVs and HPs, e.g., by shifting power consumption of EVs in peak periods to off-peak periods to provide flexibility. Firstly, each aggregator sets a bidding price for its bid according to the maximum bidding price stipulated in the FRT and historical data. A large bidding price may make the bid unaccepted in the DFM, whereas a small price may denote a small revenue. Therefore, the bidding price should be determined properly to make the bid competitive in the DFM and to enable the aggregator to gain as many revenues as possible at the same time. This paper focuses on the optimal flexibility service bid formulation under a given bidding price. The aggregator solves the following optimization model to optimally determine the amount of flexibility provided at each congestion hour, energy payback hour, and amount of payback power.

For aggregator $a$ at node $n$ with a bidding price $\lambda_{a, n}^{\text {fle }}$ :

$$
\begin{aligned}
& \operatorname{Max} \sum_{t \in T^{\mathrm{c}}}\left\{\lambda_{a, h}^{\mathrm{fle}} f_{t, a, n}^{\mathrm{fle}}-\sum_{e \in N_{a, h}^{\mathrm{ec}}} c_{t, e}^{\mathrm{ev}}\left(f_{t, e}^{\mathrm{ev}}\right)^{2}-\sum_{h \in N_{a, n}^{\mathrm{np}}} c_{t, h}^{\mathrm{hp}}\left(f_{t, h}^{\mathrm{hp}}\right)^{2}\right\}-\sum_{e \in N_{a, h}^{\mathrm{ev}}} \lambda_{e}^{\mathrm{ev}}-\sum_{h \in N_{a, h}^{\mathrm{np}}} \lambda_{h}^{\mathrm{hp}} \\
& \left\{\begin{array}{l}
f_{t, e}^{\mathrm{ev}}=\hat{p}_{t, e}^{\mathrm{ev}}-p_{t, e}^{\mathrm{ev}} ; \forall t \in T^{\mathrm{c}}, e \in N^{\mathrm{ev}} \\
f_{t, h}^{\mathrm{hp}}=\hat{p}_{t, h}^{\mathrm{hp}}-p_{t, h}^{\mathrm{ph}} ; \forall t \in T^{\mathrm{c}}, h \in N^{\mathrm{hp}} \\
f_{t, e}^{\mathrm{ev}} \geq 0, f_{t, e}^{\mathrm{ev}} \geq 0 ; \forall t \in T^{\mathrm{c}}, e \in N^{\mathrm{ev}}, h \in N^{\mathrm{hp}}
\end{array}\right.
\end{aligned}
$$

$$
\left\{\begin{array}{l}
r_{t, e}^{\mathrm{ev}}=p_{t, e}^{\mathrm{ev}}-\hat{p}_{t, e}^{\mathrm{ev}} ; \forall t \in T^{\mathrm{rb}}, e \in N^{\mathrm{ev}} \\
r_{t, h}^{\mathrm{hp}}=p_{t, h}^{\mathrm{hp}}-\hat{p}_{t, h}^{\mathrm{hp}} ; \forall t \in T^{\mathrm{rb}}, h \in N^{\mathrm{hp}} \\
r_{t, e}^{\mathrm{ev}} \geq 0, r_{t, e}^{\mathrm{ev}} \geq 0 ; \forall t \in T^{\mathrm{rb}}, e \in N^{\mathrm{ev}}, h \in N^{\mathrm{hp}}
\end{array}\right.
$$

$$
\begin{aligned}
& \left\{\begin{array}{l}
f_{t, a, n}^{\mathrm{fle}}=\sum_{e \in N_{a, n}^{\mathrm{ev}}} f_{t, e}^{\mathrm{ev}}+\sum_{h \in N_{a, n}^{\mathrm{hp}}} f_{t, h}^{\mathrm{hp}} ; \forall t \in T^{\mathrm{c}}, a \in N^{\mathrm{ag}}, n \in N^{\mathrm{nd}} \\
r_{t, a, n}^{\mathrm{rb}}=\sum_{e \in N_{a, n}^{\mathrm{ec}}}^{\mathrm{nd}} r_{t, e}^{\mathrm{ev}}+\sum_{h \in N_{a, n}^{\mathrm{hp}}} r_{t, h}^{\mathrm{hp}} ; \forall t \in T^{\mathrm{rb}}, a \in N^{\mathrm{ag}}, n \in N^{\mathrm{nd}}
\end{array}\right. \\
& \left\{\begin{array}{l}
\left|r_{t a, n}^{\mathrm{rb}}\right| \leq x_{t, a, n}^{\mathrm{rb}} M ; \forall t \in T^{\mathrm{rb}}, a \in N^{a}, n \in N^{\mathrm{nd}} \\
\sum_{t \in T^{\mathrm{rb}}}^{\mathrm{rb}} x_{t, a, n}^{\mathrm{rb}}=1 ; \forall a \in N^{a}, n \in N^{\mathrm{nd}} \\
x_{t, a, n}^{\mathrm{rb}} \in\{0,1\}
\end{array}\right. \\
& r_{t, a, n}^{\mathrm{rb}} \leq \alpha \sum_{t^{*}} f_{t^{\prime}, a, n}^{\mathrm{fle}} ; \forall t \in T^{\mathrm{rb}}, a \in N^{a}, n \in N^{\mathrm{nd}}
\end{aligned}
$$

$$
e_{e}^{\min } \leq \sum_{t_{-} \leq t}\left(p_{t_{-}, e}^{\mathrm{ev}}-d_{t_{-}, e}\right)+e_{e, t_{0}} \leq e_{e}^{\max } ; \forall t \in T, e \in N^{\mathrm{ev}}
$$




$$
\begin{aligned}
& p_{e}^{\mathrm{ev}, \min } S_{t, e} \leq p_{t, e}^{\mathrm{ev}} \leq s_{t, e} p_{e}^{\mathrm{ev}, \mathrm{max}} ; \forall t \in T, e \in N^{\mathrm{ev}} \\
& c_{h}^{\mathrm{cop}} p_{t, h}^{\mathrm{hp}}-k_{1}\left(K_{t, h}^{\mathrm{h}}-K_{t, h}^{\mathrm{u}}\right)=k_{2}\left(K_{t, h}^{\mathrm{h}}-K_{t-1, h}^{\mathrm{h}}\right) ; \forall t \in T, h \in N^{\mathrm{hp}} \\
& K_{h}^{\mathrm{h}, \mathrm{min}} \leq K_{t, h}^{\mathrm{h}} \leq K_{h}^{\mathrm{h}, \text { max }} ; \forall t \in T, h \in N^{\mathrm{hp}} \\
& p_{h}^{\mathrm{hp}, \min } \leq p_{t, h}^{\mathrm{hp}} \leq p_{h}^{\mathrm{hp}, \max } ; \forall t \in T, h \in N^{\mathrm{hp}} \\
& \left\{\begin{array}{l}
\lambda_{e}^{\mathrm{ev}}=\sum_{t \in T} \lambda_{t}^{\mathrm{DA}}\left(p_{t, e}^{\mathrm{ev}}-\hat{p}_{t, e}^{\mathrm{ev}}\right) ; \forall e \in N_{a, h}^{\mathrm{ev}} \\
\lambda_{e}^{\mathrm{hp}}=\sum_{t \in T} \lambda_{t}^{\mathrm{DA}}\left(p_{t, e}^{\mathrm{hp}}-\hat{p}_{t, e}^{\mathrm{hp}}\right) ; \forall h \in N_{a, h}^{\mathrm{hp}}
\end{array}\right.
\end{aligned}
$$

The objective function (8) is to maximize the aggregator's revenues if the bid is accepted in the DLM. The first term in (8) represents revenues of selling flexibility at the bidding price, where $f_{t, a, n}^{\text {fle }}$ is the amount of available flexibility provided by $a$-th aggregator at node $n$ at hour $t$. The second and third terms represent payments to customers for providing flexibility, where $f_{t, e}^{\mathrm{ev}}$ and $f_{t, h}^{\mathrm{hp}}$ are the amount of flexibility provided by $e$-th EV and $h$-th HP, respectively, and $c_{t, e}^{\mathrm{ev}}$ and $c_{t, h}^{\mathrm{hp}}$ are flexibility cost coefficients of $e$-th EV and $h$-HP at hour $t$, respectively. It is assumed that the payment of one unit of flexibility has a linear relation to the total amount of flexibility [5]. Each customer can adjust coefficients $c_{t, e}^{\mathrm{ev}}$ and $c_{t, h}^{\mathrm{hp}}$ to change its willingness to provide flexibility. The last two terms are to compensate customers for their increased day-ahead energy costs due to energy rescheduling, where $\lambda_{e}^{\mathrm{ev}}$ and $\lambda_{h}^{\mathrm{hp}}$ are increased day-ahead energy costs of $e$-th EV and $h$-th HP, respectively. When a customer decreases its power consumption to provide flexibility at one hour, it requires payback power at another hour. Since the baseline energy schedule has the minimum energy cost, energy rescheduling will increase the energy cost of the customer. To guarantee that customers have positive net profits when they provide flexibility, the aggregators are required to compensate customers for the increased day-ahead energy costs.

The amount of flexibility provided by each EV and HP at each congestion hour is calculated in (9), where $\hat{p}_{t, e}^{\mathrm{ev}}$ and $\hat{p}_{t, h}^{\text {hp }}$ are baseline energy schedules of $e$-th EV and $h$-th HP at hour $t$, respectively. The amount of payback power required by each $\mathrm{EV}\left(r_{t, e}^{\mathrm{ev}}\right)$ and HP $\left(r_{t, h}^{\mathrm{hp}}\right)$ at each allowable payback hour is calculated $(10)$. The total amount of flexibility $\left(f_{t, a, n}^{\mathrm{fle}}\right)$ and the total amount of payback power $\left(r_{t, a, n}^{\mathrm{rb}}\right)$ are calculated in and (11). Constraint (12) guarantees that the aggregator has payback power at one hour only, i.e., aggregator $a$ at node $n$ can have payback power at hour $t$ if $x_{a, n, t}^{\mathrm{rb}}=1$, where $M$ is a very big number. Constraint (13) represents that the amount of payback power is constrained by the maximum payback percentage $\alpha$ stipulated in the FRT. Constraints (14)-(18) are the operation constraints of EVs and HPs, as described in (2)-(6). The increased dayahead energy costs of EVs and HPs are calculated in (19).

\subsection{DFM clearing problem}

After the flexibility service bidding process, the DFM is cleared to determine the procurement percentages of flexibility service bids to resolve congestion with the minimum flexibility procurement cost. The market clearing problem is formulated as below,

$$
\begin{aligned}
& \text { Min. } \sum_{t \in T, a \in N^{\mathrm{ag}}, n \in N^{\mathrm{nd}}} \lambda_{a, n}^{\mathrm{fle}} f_{t, a, n}^{\mathrm{ag}} \\
& \sum_{l \in N^{l}} G_{n, l} F_{t, l}=p_{t, n} ; \forall t \in T, n \in N^{\mathrm{nd}} \\
& p_{t, n}=q_{t, n} \operatorname{tg}\left(\phi_{n}\right) ; \forall t \in T, n \in N^{\mathrm{nd}} \\
& \hat{p}_{t, n}=p_{t, n}+\sum_{a \in N^{\mathrm{ag}}} f_{t, a, n}^{\mathrm{ag}} ; \forall t \in T, n \in N^{\mathrm{nd}}
\end{aligned}
$$




$$
\begin{aligned}
& f_{t, a, n}^{\mathrm{ag}}=x_{t, a, n}^{\mathrm{fle}} f_{t, a, n}^{\mathrm{fle}} ; \forall t \in T, a \in N^{\mathrm{ag}}, n \in N^{\mathrm{nd}} \\
& \left|F_{t, l}\right| \leq F_{l}^{\mathrm{max}} ; \forall t \in T, l \in N^{l} \\
& \left(V^{s}\right)^{2}-\operatorname{Re}\left(\sum_{n^{n} \in N^{\text {nd }}} z_{n, n^{*}}\left(p_{t, n^{*}}-j q_{t, n^{*}}\right)\right) \geq V^{\mathrm{min}} ; \forall t \in T, n \in N^{\mathrm{nd}}
\end{aligned}
$$

The objective function (20) is to minimize the flexibility procurement cost, where $f_{t, a, n}^{\mathrm{ag}}$ is the amount of flexibility purchased from $a$-th aggregator at node $n$ at hour $t$. Constraint (21) represents the active power flow balance at each node at each hour, where $p_{t, n}$ is active power consumption at node $n$ at hour $t, F_{t, l}$ is the active power flow on $l$-th line at hour $t$, and $G_{n, l}$ is the mapping matrix. The reactive power is modeled in (22) to maintain a constant power factor at each node, where $q_{t, n}$ is the reactive power consumption at node $n$ at hour $t$, and $\cos \left(\phi_{n}\right)$ is the power factor at node $n$. Constraint (23) models the relation between baseline power consumption $\left(\hat{p}_{t, n}\right)$ and final power consumption after providing flexibility. The amount of flexibility purchased from each flexibility service bid is calculated in (24), where $x_{t, a, n}^{\text {fle }}$ is the procurement percentage of the flexibility bid provided by $a$-th aggregator at node $n$ at hour $t$. Constraint (25) represents line capacity limits, where $F_{l}^{\max }$ is the line capacity of $l$-th line. Constraint (26) is the linearized voltage magnitude constraint [34], where $V^{s}$ is the voltage magnitude at the substation node, $V^{\min }$ is the lower bound of the voltage magnitude, $\operatorname{Re}()$ is the operation to extract the real part of a complex number, and $z_{n, n^{*}}$ is the element at the $n$-th row and $n^{*}$-th column of the inverse matrix of the partial nodal admittance matrix $Y_{l l}$, which is a submatrix of the full admittance matrix $Y$ :

$$
Y=\left[\begin{array}{cc}
Y_{00} & Y_{0 l} \\
Y_{l 0} & Y_{l l}
\end{array}\right]
$$

As shown in (21)-(22) and (25)-(26), network operation constraints are incorporated into the market clearing problem in order to obtain a flexibility procurement solution that is technically feasible. Therefore, the market operator is assumed to know network parameters [17]-[20]. To relax this assumption, this paper develops an ADMM-based market clearing strategy, in which the market operator clears the market with communication with the DSO such that the market clearing solution respects network operation constraints without posing network parameters to the market operator.

\subsection{Rescheduling after flexibility activation}

After the DFM clearing, each aggregator reschedules its contractual EVs and HPs according to the amount of flexibility purchased in the DFM. The rescheduling model is the optimal flexibility bidding model in (8)-(19) with the variable $f_{t, a, n}^{\mathrm{fle}}$ fixed as the amount of flexibility purchased in the DFM. The rescheduling model is formulated as,

$$
\operatorname{Min}(8)
$$

$$
\text { Subject to: (9)-(19); } f_{t, a, n}^{\mathrm{ag}}=f_{t, a, n}^{\mathrm{fle}} ; \forall t \in T^{\mathrm{c}}, a \in N^{\mathrm{ag}}, n \in N^{\mathrm{nd}}
$$

\section{The ADMM-based Market Clearing Strategy}

In this section, an ADMM-based algorithm is developed first to solve the market clearing problem with an iterative procedure, as shown in Table 3. Then, an ADMM-based market clearing strategy is proposed based on the ADMM-based algorithm. Since the market clearing problem in (20)-(26) is formulated as a convex model, the convergence of the ADMMbased algorithm is guaranteed [22].

Table 3. Pseudo code of the ADMM-based algorithm

\begin{tabular}{ll}
\hline \hline \multicolumn{2}{l}{ ADMM-base algorithm for the market clearing model } \\
\hline 1: inputs $\quad$ Parameters of the system and flexibility bids
\end{tabular}




\begin{tabular}{ll} 
2: outputs & The amount of flexibility purchased from each flexibility service bid \\
\hline \hline 3: while & $\sum_{t \in T, a \in N^{\mathrm{a}}, n \in N^{\text {nd }}}\left(\tilde{f}_{t, a, n}^{\mathrm{ag},(k+1)}-f_{t, a, n}^{\mathrm{ag},(k+1)}\right)>\sigma_{1} ; \sum_{t \in T, a \in N^{\mathrm{a}}, n \in N^{\text {nd }}}\left(\tilde{f}_{t, a, n}^{\mathrm{ag},(k+1)}-\tilde{f}_{t, a, n}^{\mathrm{ag},(k)}\right)>\sigma_{2}$ \\
4: do & \\
5: & Solve sub-problem I to update primal variabels $\mathbf{X}$ \\
6: & Solve sub-problem II to update primal variables $\mathbf{Y}$ \\
7: & Update dual variables using (29) \\
8: end & \\
\hline \hline
\end{tabular}

\subsection{The ADMM-based algorithm}

\subsubsection{Reformulation of the market clearing model}

Before solving the market clearing model, a set of auxiliary variables $\tilde{f}_{t, a, n}^{\mathrm{ag}}$ and a set of equality constraints are introduced as below,

$$
f_{t, a, n}^{\mathrm{ag}}=\tilde{f}_{t, a, n}^{\mathrm{ag}} ; \forall t \in T, a \in N^{\mathrm{ag}}, n \in N^{\mathrm{nd}}
$$

By replacing the original variables $f_{t, a, n}^{\text {ag }}$ in (23) with auxiliary variables, the original market clearing model is reformulated as below,

Min. (20)

Subject to: (21)-(22) and (24)-(27)

$$
\hat{p}_{t, n}=p_{t, n}+\sum_{a \in N^{\mathrm{ag}}} \tilde{\hat{f}}_{t, a, n}^{\mathrm{ag}} ; \forall t \in T, n \in N^{\mathrm{nd}}
$$

\subsubsection{Augmented Lagrangian}

The augmented Lagrangian is formulated by adding the equality constraint (27) into the objective function (20) through dual variables $\mu_{t, a, n}$, as below,

$$
\text { Min. } \sum_{t \in T, a \in N^{\mathrm{ag}}, n \in N^{\mathrm{nd}}} \lambda_{a, n}^{\mathrm{fle}} f_{a, n, t}^{\mathrm{ag}}+\sum_{t \in T, a \in N^{\mathrm{ag}}, n \in N^{\mathrm{nd}}} \mu_{t, a, n}\left(\tilde{f}_{t, a, n}^{\mathrm{ag}}-f_{t, a, n}^{\mathrm{ag}}\right)+\frac{\rho}{2}\left\|\tilde{f}_{t, a, n}^{\mathrm{ag}}-f_{t, a, n}^{\mathrm{ag}}\right\|^{2}
$$

Subject to: (21)-(22) and (24)-(26), (28)

The augmented Lagrangian is optimized over two groups of primal variables $(\mathbf{X}, \mathbf{Y})$ and one group of dual variables $(\boldsymbol{\Lambda})$, as defined below,

$$
\left\{\begin{array}{l}
\mathbf{X}=\left[\tilde{f}_{t, a, n}^{\mathrm{ag}}, p_{t, n}, q_{t, n}, F_{t, l}\right] \\
\mathbf{Y}=\left[f_{t, a, n}^{a g}, x_{t, a, n}^{\mathrm{fle}}\right] \\
\boldsymbol{\Lambda}=\left[\mu_{t, a, n}\right]
\end{array}\right.
$$

\subsubsection{Iterative procedure}

In the ADMM-based algorithm, primal variables and dual variables of the augmented Lagrangian are optimized with an iterative procedure. Two groups of primal variables are optimized in sub-problems I and II, respectively.

(1) Sub-problem I

In the sub-problem I, at the (k+1)-th iteration, primal variables $\mathbf{X}^{k+1}$ are optimized with the values of $\mathbf{Y}^{*}$ and $\boldsymbol{\Lambda}^{*}$ obtained at the $k$-th iteration. The sub-problem I is formulated as below,

$$
\text { Min. } \sum_{t \in T, a \in N^{\mathrm{ag}}, n \in N^{\mathrm{nd}}} \mu_{t, a, n}^{(k)} \tilde{f}_{t, a, n}^{\mathrm{ag},(k+1)}+\frac{\rho}{2}\left\|\tilde{f}_{t, a, n}^{\mathrm{ag},(k+1)}-f_{t, a, n}^{\mathrm{ag},(k)}\right\|^{2} ;
$$

Subject to: (21)-(22), (25)-(26), and (28) 
(2) Sub-problem II

In the sub-problem II, primal variables $\mathbf{Y}^{k+1}$ are optimized at $(k+1)$-th iteration with the values of $\mathbf{X}^{*}$ and $\mathbf{\Lambda}^{*}$ obtained at the $k$-th iteration. The sub-problem II is formulated as below,

$$
\text { Min. } \sum_{t \in T, a \in N^{\mathrm{ag}}, n \in N^{\mathrm{nd}}} \lambda_{a, n}^{\mathrm{fle}} f_{t, a, n}^{\mathrm{ag},(k+1)}-\sum_{t \in T, a \in N^{\mathrm{as}}, n \in N^{\mathrm{nd}}} \mu_{t, a, n}^{(k)} f_{t, a, n}^{\mathrm{ag},(k+1)}+\frac{\rho}{2}\left\|\tilde{f}_{t, a, n}^{\mathrm{ag},(k)}-f_{t, a, n}^{\mathrm{ag},(k+1)}\right\|^{2} ;
$$

Subject to: (24)

(3) Update of dual variables

After solving sub-problems I and II, dual variables are updated using the values of $\mathbf{X}^{*}$ and $\mathbf{Y}^{*}$ obtained at $(k+1)$-th iteration in (29).

$$
\mu_{t, a, n}^{(k+1)}=\mu_{t, a, n}^{(k)}+\rho\left(\tilde{f}_{t, a, n}^{\mathrm{ag},(k+1)}-f_{t, a, n}^{\mathrm{ag},(k+1)}\right) ; \forall t \in T, a \in N^{\mathrm{ag}}, n \in N^{\mathrm{nd}}
$$

\section{(4) Convergence Criteria}

After optimizing primal variables and updating dual variables, the convergence conditions are checked. The optimizations of primal variables and update of dual variables are carried out iteratively until the aggregated primal and dual residuals (cr and cd) are lower than the specified thresholds, as shown in (30).

$$
\left\{\begin{array}{l}
\text { primal residual:cr }=\left|\sum_{t \in T, a \in N^{a}, n \in N^{\text {nd }}}\left(\tilde{f}_{t, a, n}^{\text {ag, },(k+1)}-f_{t, a, n}^{\mathrm{ag},(k+1)}\right)\right| \leq \sigma_{1} ; \\
\text { dual residual:cd }=\left|\sum_{t \in T, a \in N^{a}, n \in N^{\text {nd }}}\left(\tilde{f}_{t, a, n}^{\mathrm{ag},(k+1)}-\tilde{f}_{t, a, n}^{\mathrm{ag},(k)}\right)\right| \leq \sigma_{2} ;
\end{array}\right.
$$

(5) Dynamic update of the penalty parameter

During the iteration procedure, the penalty parameter $\rho$ is updated at each iteration according to primal and dual residuals using equation (31) [22], [35], where $\zeta$ and $\tau$ are update parameters.

$$
\rho^{k+1}= \begin{cases}\tau \rho^{k} & \text { if } \mathrm{cr} \geq \zeta \mathrm{cd} \\ \rho^{k} / \tau & \text { if } \mathrm{cd} \geq \zeta \mathrm{cr} \\ \rho^{k} & \text { otherwise }\end{cases}
$$

\subsection{The ADMM-based DFM clearing strategy}

Based on the proposed ADMM-based algorithm, the proposed DFM clearing strategy is shown in Fig. 3, where the market operator clears the market with communication with the DSO. In the interactive procedure, the parameters associated with the amount of purchased flexibility are exchanged between the market operator and the DSO. The market operator solves the subproblem II to select flexibility service bids and calculate flexibility procurement costs, while the DSO solves the sub-problem I to check if network operation constraints are violated with the selected flexibility service bids. In the proposed strategy, the market clearing solution respects network operation constraints without revealing network parameters to the market operator.

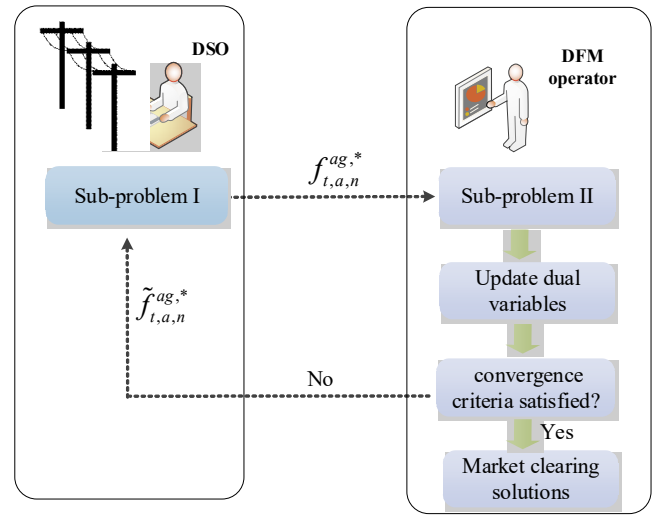

Fig. 3 The ADMM-based DFM clearing strategy 


\section{Case Studies}

Case studies were conducted on the Bus 4 distribution network of the Roy Billinton Test System (RBTS) [36] to demonstrate the effectiveness of day-ahead congestion management with the DFM. The single line diagram of the Bus 4 distribution network is shown in Fig. 4. Line segments of feeder 1 are labeled as L1-L12 and load points are labeled as LP ${ }_{1-7}$, $\mathrm{LP}_{11-16}, \mathrm{LP}_{18-25}$, and $\mathrm{LP}_{32-38}$. The data of conventional load consumption profile and lines can be found in [7]. The loading limits of L2 and L7, load point data, and key parameters of the EV and HP are listed in Table 4a-4b. Each residential load point has 200 customers, each of which owns an EV and HP to provide flexibility. It is assumed that there are four aggregators $\left(a g_{1}, a g_{2}\right.$, $\left.a g_{3}, a g_{4}\right)$ participating in the DFM. At each residential load point, each of $a g_{1}$ and $a g_{4}$ has contracts with 40 customers, and each of $a g_{2}$ and $a g_{3}$ has contracts with 60 customers. The forecasted day-ahead spot price profile is shown in Fig. 5. The lower limit of the voltage magnitude is set to be 0.95 p.u. is order to have a security margin of 0.01 p.u. compared to the assumed physical limit of 0.94 p.u. It is assumed that the bidding prices of aggregators and cost coefficients of EVs and HPs are produced randomly with the normal distribution.

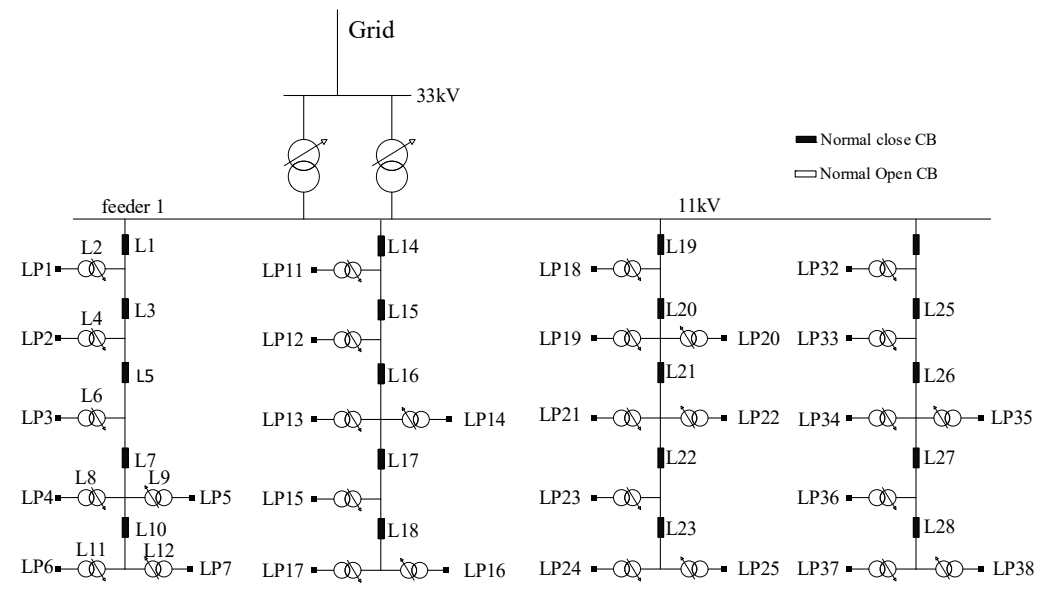

Fig. 4 The single diagram of the Bus 4 distribution network

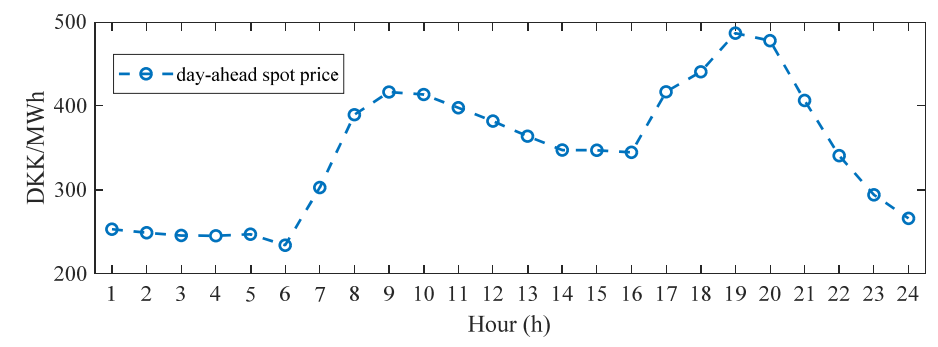

Fig. 5 Forecasted day-ahead spot price profile

Table 4a. Key parameters

\begin{tabular}{ll}
\hline \hline Parameters & Value \\
\hline COP of HP & 3.0 \\
\hline$k_{1}$ (House Type I-V) & $0.280 / 0.315 / 0.350 / 0.385 / 0.420$ \\
\hline$k_{2}$ (House Type I-V) & $3.638 / 4.093 / 4.548 / 5.002 / 5.457$ \\
\hline Lower and upper limits of SOC & $20 \%-90 \%$ \\
\hline Thermal comfort range & $20-24^{\circ} \mathrm{C}$ \\
\hline Peak consumption power & $6 \mathrm{~kW}$ \\
\hline EV battery size & $30 \mathrm{kWh}$ \\
\hline Energy consumption per $\mathrm{km}$ & $150 \mathrm{kWh} / \mathrm{km}$ \\
\hline Peak charging power & $11 \mathrm{~kW}$ \\
\hline Loading limit of L2/L7 & $1900 / 4100 \mathrm{~kW}$ \\
\hline Resistance/reactance & $0.26 / 0.027 \mathrm{omh} / \mathrm{km}$ \\
\hline \hline
\end{tabular}


Table 4b. Load point data

\begin{tabular}{c|c|c|c}
\hline \hline load points $(\mathbf{L P})$ & types of customers & peak conv. load $[\mathbf{k W}]$ & number of customers per LP \\
\hline $\mathrm{LP}_{1-4}, \mathrm{LP}_{11-13}, \mathrm{LP}_{18-21}, \mathrm{LP}_{32-35}$ & residential & 886.9 & 200 \\
$\mathrm{LP}_{5}, \mathrm{LP}_{14-15}, \mathrm{LP}_{22}, \mathrm{LP}_{36}$ & residential & 813.7 & 200 \\
$\mathrm{LP}_{23}, \mathrm{LP}_{37}$ & residential & 986.9 & 200 \\
$\mathrm{LP}_{6-7}, \mathrm{LP}_{16-17}$, & commercial & 415.0 & 10 \\
$\mathrm{LP}_{24-25}, \mathrm{LP}_{38}$ & commercial & 986.9 & 10 \\
\hline \hline
\end{tabular}

Based on the forecasted day-ahead spot prices, the energy suppliers obtain baseline day-ahead energy schedules and submit the schedules to the DSO for technical validation. The DSO conducts the power flow analysis to identify if the line capacity and voltage magnitude constraints are violated. As shown in Fig. 6, there is an overload of $132.8 \mathrm{~kW}$ at L2 at $t_{6}$, and there are overloads of $378.2 \mathrm{~kW}$ and $86.1 \mathrm{~kW}$ at L7 at $t_{6}$ and $t_{18}$, respectively. As shown in Fig. 7, voltage magnitudes at load points $\mathrm{LP}_{23}$ and $\mathrm{LP}_{37}$ are below the lower limit at $t_{6}$. To resolve these problems, the DSO needs to procure flexibility in the DFM to perform overload and voltage management. After conducting the power flow analysis and voltage sensitivity analysis, the DSO submits a FRT shown in Table 5 to the DFM. In the study, the voltage sensitivity with respect to nodal power injections is derived using the linearized voltage equation in (26). The sensitivity of the voltage magnitude at $i$-th node with respect to the active power injection at $j$-th node is calculated below,

$$
\frac{\partial V_{t, i}}{\partial p_{t, j}}=-\left(r_{i, j}+x_{i, j} \frac{1}{\operatorname{tg}\left(\phi_{j}\right)}\right) ; \forall t \in T, i, j \in N^{\mathrm{nd}}
$$

where $r_{i, j}$ and $x_{i, j}$ are the resistance and reactance of the line between nodes $i$ and $j$, respectively. According to the voltage sensitivity calculation and voltage magnitude deviation from the lower limit, the approximate values of minimum and maximum amount of flexibility required at $t_{6}$ for voltage management can be obtained.

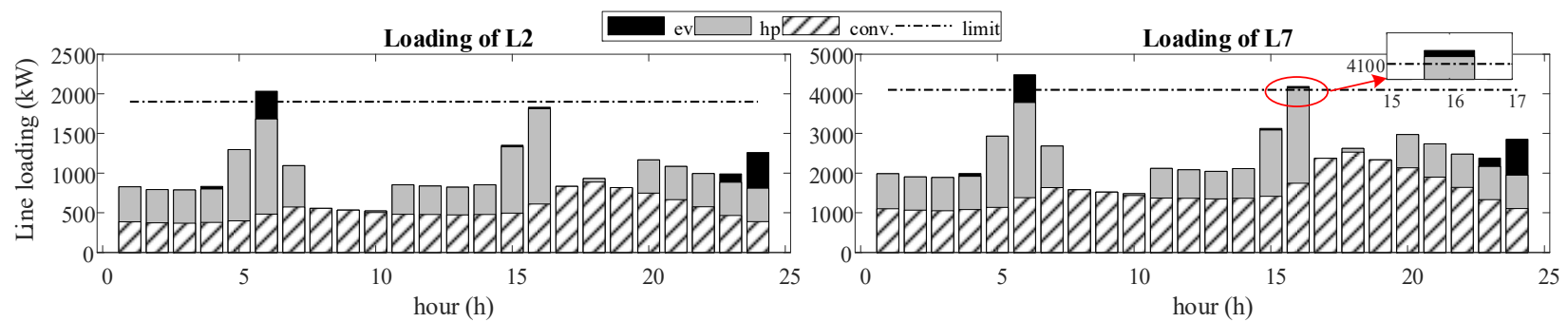

Fig. 6 Line loadings of L2 and L7

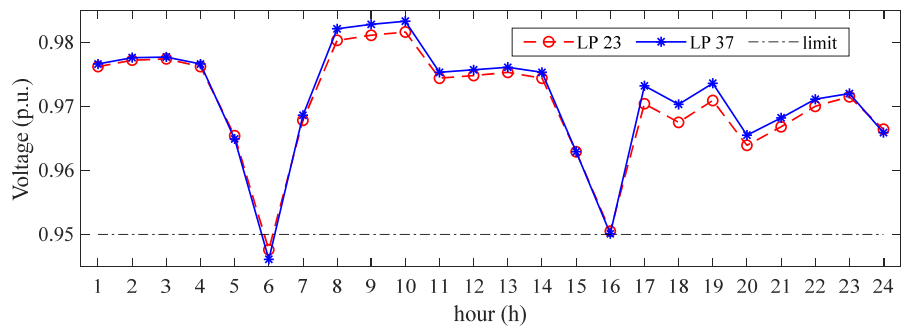

Fig. 7 Voltage magnitudes at $\mathrm{LP}_{23}$ and $\mathrm{LP}_{37}$

Table 5. Flexibility requirement table

\begin{tabular}{c|c|c|c|c|c}
\hline \hline \multirow{2}{*}{ load points } & \multicolumn{2}{|c|}{$\begin{array}{c}\text { minimum amount of flexibility required } \\
{[\mathrm{kW}]}\end{array}$} & \multirow{2}{*}{ payback hours } & $\begin{array}{c}\text { upper limit on } \\
\text { payback percentage }\end{array}$ & $\begin{array}{c}\text { maximum price } \\
{[\mathrm{DKK} / \mathrm{kW}]}\end{array}$ \\
\cline { 2 - 5 } & $t_{6}$ & $t_{16}$ & $t_{1-5, t 7-15, t_{17-24}}$ & $150 \%$ & 1.5 \\
\hline $\mathrm{LP}_{1}$ & 132.8 & - & & \\
\hline \hline
\end{tabular}




\begin{tabular}{c|c|c|c|c|}
\hline \hline $\mathrm{LP}_{4-5}$ & 378.2 & 86.1 & $t_{1-5}, t_{7-15}, t_{17-24}$ & \multirow{2}{*}{} \\
\cline { 1 - 4 } $\mathrm{LP}_{18-23}$ & 317.6 & - & $t_{1-5}, t_{7-15}, t_{17-24}$ & \\
\cline { 1 - 3 } $\mathrm{LP}_{32-37}$ & 433.4 & - & $t_{1-5}, t_{7-15}, t_{17-24}$ & \\
\hline \hline
\end{tabular}

\subsection{Feeder overload management}

Upon the flexibility request, each aggregator solves the optimal flexibility bidding model to formulate flexibility service bids according to the FRT. As listed in Table 6, four aggregators provide flexibility service bids at $\mathrm{LP}_{1}$ and $\mathrm{LP}_{4-5}$ that the DSO can use to resolve congestion on L2 and L7. Specifically, at $\mathrm{LP}_{1}$, four aggregators $\left(a g_{1}-a g_{4}\right)$ provide flexibility at $t_{6}$ and choose to have payback power at $t_{4}$. At $\mathrm{LP}_{4}, a g_{1}-a g_{4}$ provide flexibility at $t_{6}$ and $t_{16}$ simultaneously and require payback power at $t_{10}$, however, at $\mathrm{LP}_{5}, a g_{2}-a g_{4}$ provide flexibility at $t_{6}$ only. After the flexibility service bidding process, the market operator clears the DFM to obtain the procurement percentage of each bid, as listed in Table 7. With the market clearing solution, the aggregators reschedule EVs and HPs to provide committed flexibility and formulate the final day-ahead energy schedules. The resulting loadings of L2 and L7 are shown in Fig. 8. It can be seen that congestion on L2 and L7 is resolved and energy payback occurs at $t_{4}$ and $t_{10}$ without causing new congestion, which demonstrates the effectiveness of overload management with the DFM.

Table 6. Flexibility service bids

\begin{tabular}{|c|c|c|c|c|c|c|}
\hline \multirow{2}{*}{ aggregator } & \multirow{2}{*}{$\begin{array}{c}\text { price } \\
{[\mathrm{DKK} / \mathrm{kW}]}\end{array}$} & \multirow{2}{*}{ load point } & \multicolumn{2}{|c|}{ flexibility amount [kW] } & \multicolumn{2}{|c|}{ payback hour and amount [kW] } \\
\hline & & & $t_{6}$ & $t_{16}$ & $t_{4}$ & $t_{10}$ \\
\hline \multirow{3}{*}{$a g_{1}$} & 0.75 & $\mathrm{LP}_{1}$ & 60.896 & - & 66.478 & - \\
\hline & 0.62 & $\mathrm{LP}_{4}$ & 49.673 & 20.819 & - & 74.341 \\
\hline & 0.70 & $\mathrm{LP}_{5}$ & 52.857 & 22.692 & - & 79.820 \\
\hline \multirow{3}{*}{$a g_{2}$} & 0.58 & $\mathrm{LP}_{1}$ & 87.408 & - & 94.763 & - \\
\hline & 0.66 & $\mathrm{LP}_{4}$ & 78.673 & 34.506 & - & 119.152 \\
\hline & 0.63 & $\mathrm{LP}_{5}$ & 89.463 & & 97.047 & \\
\hline \multirow{3}{*}{$a g_{3}$} & 0.53 & $\mathrm{LP}_{1}$ & 83.661 & - & 90.853 & - \\
\hline & 0.71 & $\mathrm{LP}_{4}$ & 81.833 & 35.373 & - & 123.723 \\
\hline & 0.59 & $\mathrm{LP}_{5}$ & 84.901 & - & 92.302 & \\
\hline \multirow{3}{*}{$a g_{4}$} & 0.84 & $\mathrm{LP}_{1}$ & 67.318 & - & 73.219 & - \\
\hline & 0.78 & $\mathrm{LP}_{4}$ & 57.224 & 24.412 & - & 85.806 \\
\hline & 0.73 & $\mathrm{LP}_{5}$ & 54.810 & - & 59.565 & - \\
\hline
\end{tabular}

Table 7. Procurement percentages of flexibility service bids and total amount of purchased flexibility

\begin{tabular}{c|c|c|c}
\hline \hline \multirow{2}{*}{ aggregator } & \multirow{2}{*}{ load point } & \multicolumn{2}{|c}{ percentage } \\
\cline { 2 - 4 } & & $t_{6}$ & $t_{16}$ \\
\hline \multirow{3}{*}{$a g_{1}$} & $\mathrm{LP}_{1}$ & - & - \\
\cline { 2 - 4 } & $\mathrm{LP}_{4}$ & 1.000 & 1.000 \\
\cline { 2 - 4 } & $\mathrm{LP}_{5}$ & 0.392 & 1.000 \\
\hline \multirow{4}{*}{$a g_{2}$} & $\mathrm{LP}_{1}$ & 0.562 & - \\
\cline { 2 - 4 } & $\mathrm{LP}_{4}$ & 1.000 & 1.000 \\
\cline { 2 - 4 } & $\mathrm{LP}_{5}$ & 1.000 & - \\
\hline \multirow{3}{*}{$a g_{3}$} & $\mathrm{LP}_{1}$ & 1.000 & - \\
\cline { 2 - 4 } & $\mathrm{LP}_{4}$ & - & - \\
\cline { 2 - 4 } & $\mathrm{LP}_{5}$ & 1.000 & - \\
\hline \multirow{3}{*}{$a g_{4}$} & $\mathrm{LP}_{1}$ & - & - \\
\cline { 2 - 4 } & $\mathrm{LP}_{4}$ & - & - \\
\cline { 2 - 4 } & $\mathrm{LP}_{5}$ & 1.000 & - \\
\hline total amount $[\mathrm{kW}]$ & & 511.040 & \multicolumn{2}{|c}{} \\
\hline \hline
\end{tabular}




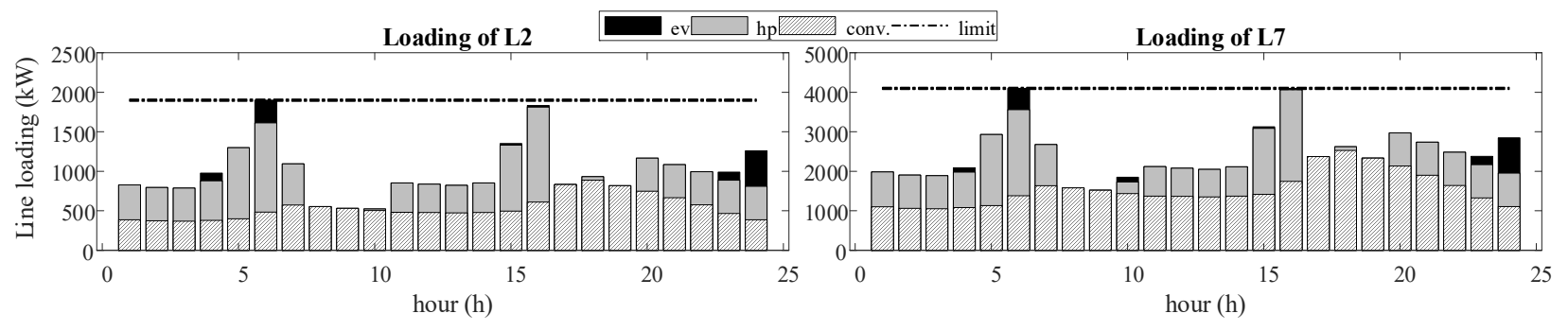

Fig. 8 Line loadings of L2 and L7 of the final day-ahead energy schedules

The baseline and final charging power profiles and SOC levels of an EV at $\mathrm{LP}_{4}$ are shown in Fig. 9. In order to provide flexibility at $t_{6}$ and $t_{16}$, the EV charges more power at $t_{10}$ so that it can have power consumption reductions at $t_{6}$ and $t_{16}$ while maintaining the sufficient SOC level for the daily driving consumption. The baseline and final power consumption profiles of a $\mathrm{HP}$ at $\mathrm{LP}_{4}$ and its household inside temperature are shown in Fig. 10. Similarly, the HP decreases power consumption at $t_{6}$ and $t_{16}$ to provide flexibility while having payback power at $t_{10}$ to maintain the household inside temperature within the thermal comfort range.
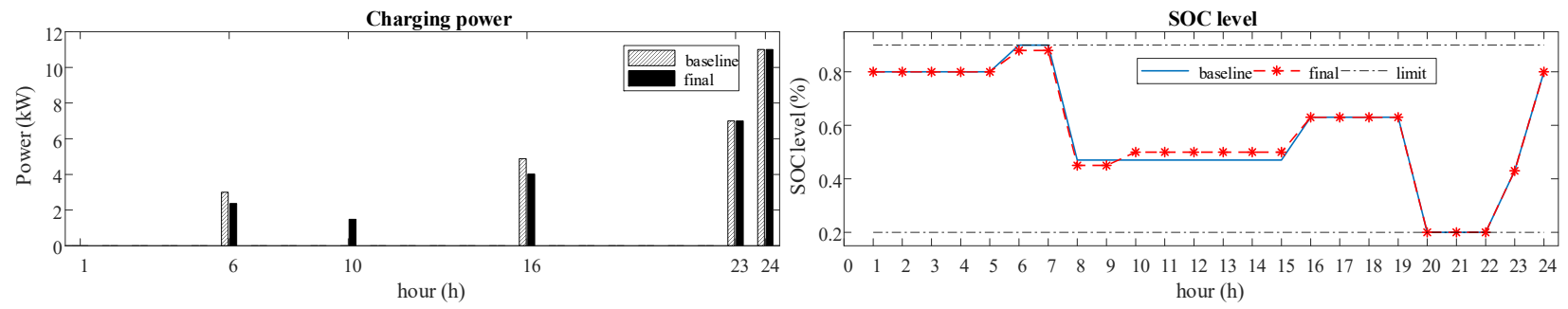

Fig. 9 Baseline and final charging power profiles and SOC levels of an EV at $\mathrm{LP}_{4}$

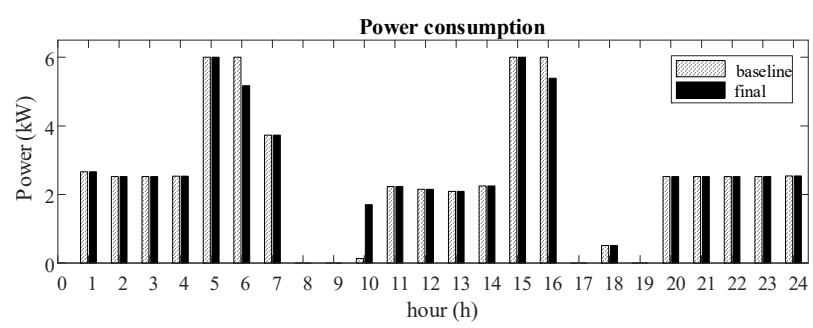

Fig. 10 Baseline and final power consumption profiles of an $\mathrm{HP}$ at $\mathrm{LP}_{4}$ and household inside temperature

\subsection{Feeder voltage management}

To perform feeder voltage management, the aggregators formulate flexibility service bids at residential loads $\mathrm{LP}_{18-23}$ and $\mathrm{LP}_{32-37}$. The procurement percentages of the bids accepted in the DFM are listed in Table 8. It is shown that four aggregators provide flexibility at $t_{6}$ and have payback power at $t_{4}$. The flexibility of $879.208 \mathrm{~kW}$ is purchased in the DFM to resolve undervoltage issues. After rescheduling EVs and HPs, the resulting voltage profiles of $\mathrm{LP}_{23}$ and $\mathrm{LP}_{37}$ are shown in Fig. 11. It is shown that the voltage magnitudes at $\mathrm{LP}_{23}$ and $\mathrm{LP}_{37}$ are above the lower limit at $t_{6}$ and voltage magnitudes at $t_{4}$ decrease due to energy payback. In the day-head scheduling horizon, voltage magnitudes and line capacity constraints are respected. Therefore, the proposed FLM is effective to perform voltage management of distribution networks.

In addition, it is important to analyze the voltage linearization error to ensure the voltage security of the market clearing solution. It is shown in Fig. 12 that the maximum difference between the accurate and approximated voltage magnitudes at all load points is 0.00266 p.u. and smaller than the preset security margin of 0.01 p.u.

Table 8. Accepted flexibility service bids and procurement percentage

\begin{tabular}{c|c|c|c|c|c}
\hline \hline \multirow{3}{*}{ aggregator } & \multirow{2}{*}{$\begin{array}{c}\text { price } \\
{[\mathrm{DKK} / \mathrm{kW}]}\end{array}$} & \multirow{2}{*}{$\begin{array}{c}\text { load } \\
\text { points }\end{array}$} & $\begin{array}{c}\text { flexibility amount } \\
{[\mathrm{kW}]}\end{array}$ & $\begin{array}{c}\text { payback hour and } \\
\text { power }[\mathrm{kW}]\end{array}$ & \multirow{2}{*}{$\begin{array}{c}\text { procurement } \\
\text { percentage }\end{array}$} \\
\cline { 4 - 6 } & & & $t_{6}$ & $t_{4}$ & 1.000 \\
\hline \multirow{2}{*}{$a g_{1}$} & 0.69 & $\mathrm{LP}_{23}$ & 59.803 & 65.197 & 1.000 \\
\cline { 2 - 5 } & 0.72 & $\mathrm{LP}_{37}$ & 59.442 & 64.848 & \\
\hline \hline
\end{tabular}




\begin{tabular}{c|c|c|c|c|c}
\hline \hline \multirow{4}{*}{$a g_{2}$} & 0.57 & $\mathrm{LP}_{21}$ & 87.923 & 95.343 & 0.372 \\
\cline { 2 - 6 } & 0.68 & $\mathrm{LP}_{23}$ & 92.910 & 100.609 & 1.000 \\
\cline { 2 - 6 } & 0.61 & $\mathrm{LP}_{36}$ & 88.942 & 96.415 & 1.000 \\
\cline { 2 - 6 } & 0.70 & $\mathrm{LP}_{37}$ & 92.393 & 100.338 & 1.000 \\
\hline \multirow{4}{*}{$a g_{3}$} & 0.62 & $\mathrm{LP}_{23}$ & 88.493 & 96.229 & 1.000 \\
\cline { 2 - 6 } & 0.76 & $\mathrm{LP}_{36}$ & 94.697 & 102.933 & 1.03 \\
\cline { 2 - 6 } & 0.74 & $\mathrm{LP}_{37}$ & 95.096 & 63.457 & 1.000 \\
\hline \multirow{3}{*}{$a g_{4}$} & 0.63 & $\mathrm{LP}_{23}$ & 58.598 & 66.258 & 1.000 \\
\cline { 2 - 6 } & 0.66 & $\mathrm{LP}_{36}$ & 60.909 & 71.658 & 1.000 \\
\cline { 2 - 6 } & 0.83 & $\mathrm{LP}_{37}$ & 66.063 & & \\
\hline
\end{tabular}
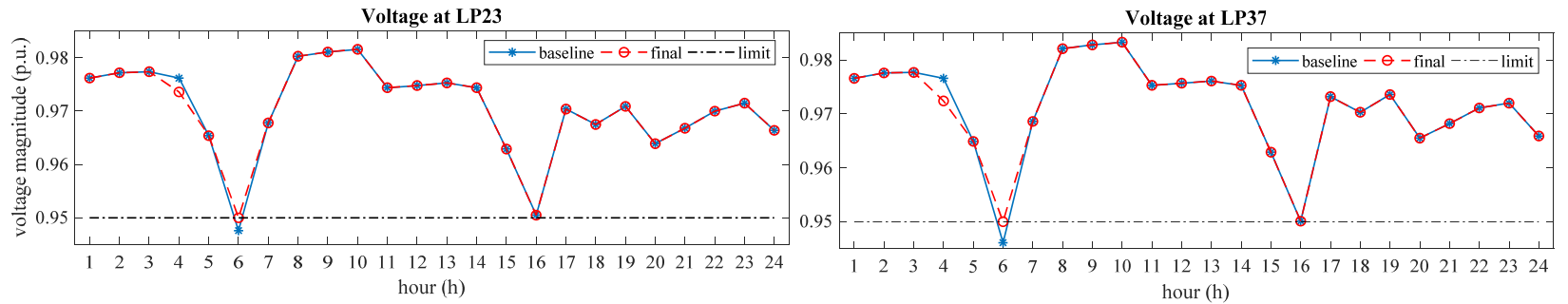

Fig. 11 Voltage magnitudes at $\mathrm{LP}_{23}$ and $\mathrm{LP}_{37}$

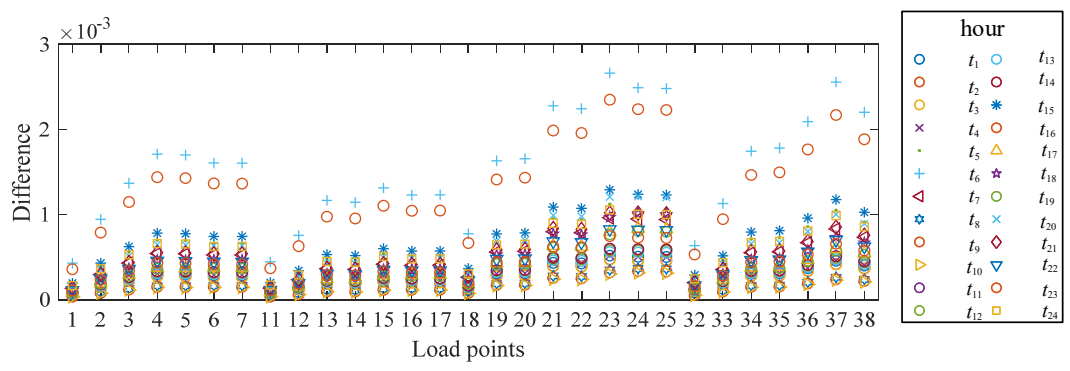

Fig. 12 Differences between the accurate and approximated voltage magnitudes

The charging power profiles and SOC levels of two EVs $\left(\mathrm{ev}_{1}\right.$ and $\left.\mathrm{ev}_{2}\right)$ at $\mathrm{LP}_{23}$ are shown in Fig. 13 to illustrate the impact of the cost coefficient on providing flexibility. Two EVs have the same driving patterns with different cost coefficients. Since $\mathrm{ev}_{1}$ has a smaller cost coefficient (0.38) than $\mathrm{ev}_{2}(0.44)$, the aggregator tends to utilize flexibility from ev 1 in order to gain more revenues. It is shown in Fig. 13 that ev $_{1}$ provides more flexibility at $t_{6}$ and needs more payback power at $t_{4}$ to maintain a sufficient SOC level for driving consumption.
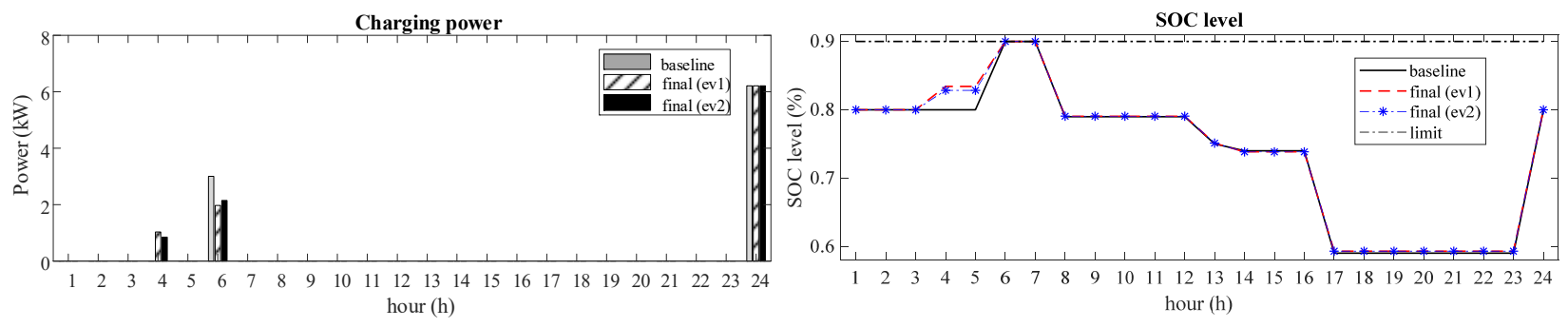

Fig. 13 Charging power profiles and SOC levels of two EVs at $\mathrm{LP}_{23}$

\subsection{Effectiveness of the proposed ADMM-based algorithm}

The market clearing problem is solved by the proposed ADMM-based algorithm in an iterative manner. In the study, the primal and dual convergence thresholds are set as 0.0001 , the initial penalty parameter is set as 10 , and update parameters $\zeta$ and $\tau$ are set as 10 and 2, respectively. As shown in Fig. 14, the aggregated primal and dual residuals can converge to the specified thresholds after 172 iterations. The iteration process of the objective value is shown in Fig. 15, which demonstrates that the ADMM-based algorithm can reproduce the centralized solution. The centralized solution is obtained by directly solving the market clearing model with the CPLEX solver. The computation times of the centralized and ADMM-based methods are presented in Table 9. Although the ADMM-based method needs more computation time than the centralized method, it can 
maintain privacy information protection, i.e., the market operator does not need to have access to system parameters. Since the flexibility market clearing is implemented in the day-ahead time frame, the computation time of a few minutes is not a big concern.

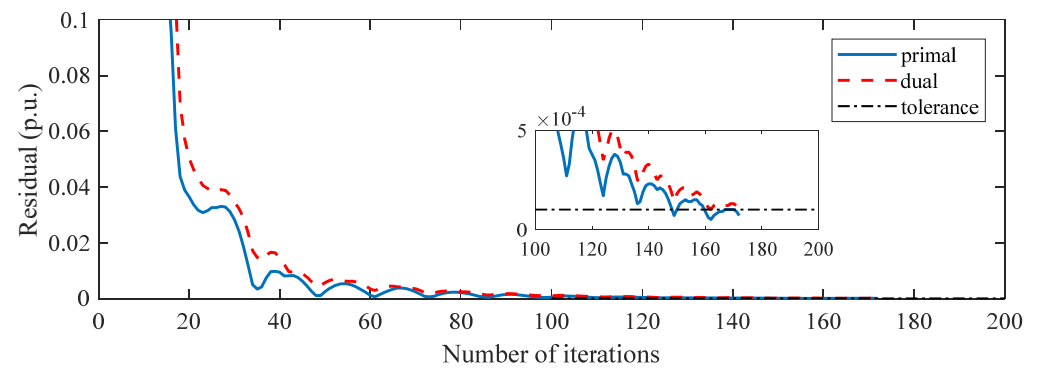

Fig. 14 Convergence process of the ADMM-based algorithm

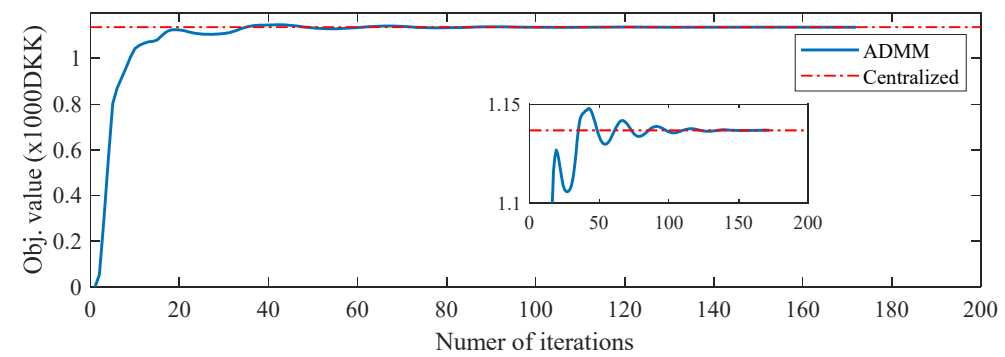

Fig. 15 Convergence of the objective value

Table 9. Computation times of the centralized and ADMM-based methods

\begin{tabular}{lcc}
\hline \hline solution method & Centralized & ADMM-based \\
\hline computation time (second) & 33 & 238 \\
\hline \hline
\end{tabular}

\subsection{Financial settlement}

The last process of the DFM framework is to carry out the financial settlement. In the proposed DFM framework, the DSO pays for the flexibility procurement while the aggregator and customers receive revenues for providing flexibility. Table 10 lists the flexibility procurement cost of the DSO and revenues received by the aggregators and customers. As shown in the capital flow in Fig. 16, customers receive payments from the aggregators because of providing flexibility and increase dayahead energy costs. Therefore, customers do not need to pay extra money for the increased energy costs and always have revenues when they provide flexibility. The aggregators receive flexibility selling revenues from the market operator or from the DSO directly and pay customers for providing flexibility and increased energy costs. Since the flexibility service bid is formulated with the maximization of the non-negative revenue of the aggregator, it ensures that the aggregator always has revenues as long as its bid is accepted. Therefore, the proposed DFM is an attractive flexibility trading platform because it is profitable for the aggregators and customers.

Table 10. Results of financial settlement

\begin{tabular}{c|c|c|c|c|c|c}
\hline \hline \multirow{2}{*}{ players } & \multirow{2}{*}{ costs of the DSO } & \multicolumn{4}{|c|}{ revenues of aggregators } & \multirow{2}{*}{ revenues of customers } \\
\cline { 3 - 6 } & & $a g_{1}$ & $a g_{2}$ & $a g_{3}$ & $a g_{4}$ & \\
\hline DKK & 1136.741 & 85.256 & 215.666 & 171.263 & 91.639 & 498.984 \\
\hline \hline
\end{tabular}




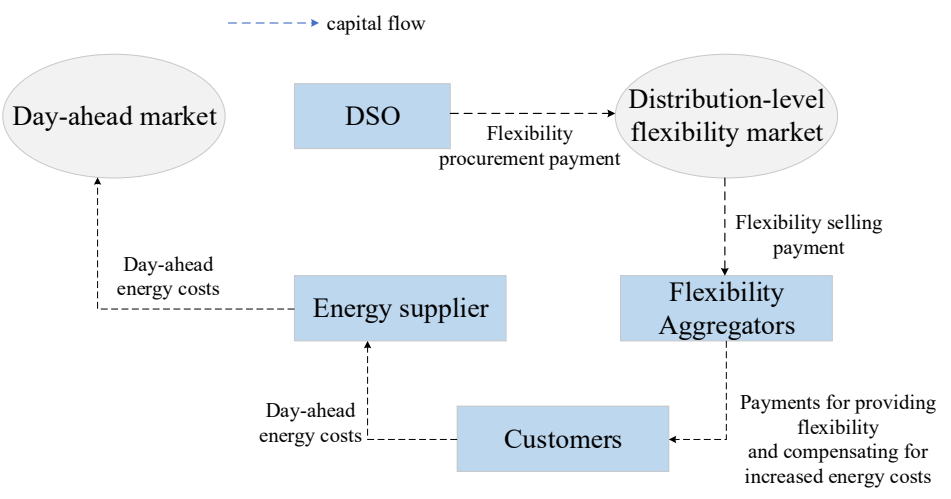

Fig. 16 Capital flow of financial settlement

\section{Conclusions}

To improve the privacy information protection of the DFM framework, this paper proposes an ADMM-based market clearing strategy, in which the market clearing problem is formulated as a linear programming model, and decomposed and solved by the ADMM-based algorithm. Based on the ADMM-based algorithm, the market operator clears the market with network operation constraints respected and without revealing network parameters to the market operator. In addition, an optimal flexibility bidding strategy for the aggregator is proposed and formulated as a MIQP model, which determines the energy payback conditions of flexibility resources considering operational constraints and enables the aggregator to receive the maximum revenue with the flexibility cost included. The case studies results demonstrate that the proposed DFM framework can perform effective day-ahead congestion management including overload and voltage management. The proposed ADMM-based market clearing strategy can efficiently solve the market clearing problem. Although it needs more computation time than the centralized strategy, it provides privacy information protection. In addition, the optimal flexibility bidding strategy guarantees that the aggregator receives the maximum revenue with the flexibility cost included, if its bid is accepted in the DFM, which incentives aggregators to gather flexibility from end-users and trade flexibility in the DFM.

The uncertainty modeling of flexible demands and renewable energy resources in the DFM framework and the mechanism of setting bidding prices for flexibility services bids will be studied in the future work.

\section{Reference}

[1] The Danish Government, Feb. 2011, Energy strategy 2050 -from coal, oil and gas to green energy. Copenhagen, Denmark, accessed on Aug. 4, 2014.

[2] P. Hallberg, et al., "Active distribution system management-A key tool for the smooth integration of distributed generation”, Eurelectric, Brussels, Belgium, Feb. 2013.

[3] Spiliotis K, Ramos Gutierrez AI, and Belmans R., "Demand flexibility versus physical network expansions in distribution grids", Applied Energy, vol. 182, pp. 613-624, Nov. 2016.

[4] U.S. Department of Energy, "Benefit of demand response in electricity market and recommendations for achieving them," Feb. 2006.

[5] F. Shen, Q. Wu, S. Huang, X. Chen, H. Liu, and Y. Xu, "Two-tier demand response with flexible demand swap and transactive control for real-time congestion management in distribution networks", Int. J. Elect. Power and Energy Syst., in press.

[6] R. Li, Q. Wu, and S. S. Oren, "Distribution locational marginal pricing for optimal electric vehicle charging management," IEEE Trans. Power Syst. vol. 29, no. 1, pp. 203-211, Jan. 2014.

[7] S. Huang, Q. Wu, S. S. Oren, R. Li, and Z. Liu, "Distribution locational marginal pricing through quadratic programming for congestion management in distribution network," IEEE Trans. Power Sys., vol. 30, no. 4, pp. 2170-2178, Jul. 2015.

[8] S. Huang, Q. Wu, L. Cheng, Z. Liu, and H. Zhao, "Uncertainty management of dynamic tariff method for congestion management in distribution networks," IEEE Trans. Power Syst., vol.31, no.6, pp. 4340-4347, Nov. 2016.

[9] S. Hanif, H. B. Gooi, T. Massier, T. Hamacher, and T. Reindl, "Distributed congestion management of distribution grids under robust 
flexible buildings operations,” IEEE Trans. Power Syst., vol. 32, no. 6, pp. 4600-4613, Nov. 2017.

[10] S. Huang, Q. Wu, H. Zhao, and C. Li, "Distributed Optimization based Dynamic Tariff for Congestion Management in Distribution Networks,” IEEE Trans. Smart Grid, vol. 10, no. 1, pp. 184-192, Jan. 2019.

[11] B. Biegel, P. Andersen, J. Stoustrup, and J. Bendtsen, "Congestion management in a smart grid via shadow prices," in Proc. 2012 8th IFAC Symposium on Power Plant and Power Syst. Control, pp. 518-523.

[12] S. Huang and Q. Wu, "Dynamic subsidy method for congestion management in distribution networks," IEEE Trans. Smart Grid, vol. 9, no. 3, May 2018.

[13] T. Morstyn, A. Teyteloym, and M. D. McCulloch, “Designing Decentralized Markets for Distribution System Flexibility”, IEEE Trans. Power Syst., vol. 34, no. 3, May 2019.

[14] D. T. Nguyen, M. Negnevitsky, and M. de Groot, "Pool-based demand response exchange-concept and modeling," IEEE Trans. Power Syst., vol. 26, no. 3, pp. 1677-1685, Aug. 2011.

[15] S. S. Torbaghan, N. Blaauwbroek, D. Kuiken, M. Gibescu, M. Hajighasemi, P. Nguyen, et al, "A market-based framework for demand side flexibility scheduling and dispatching”, Sustain Energy, Grids Networks, vol. 14, pp. 47-61, Jun. 2018.

[16] Torbaghan SS, Blaauwbroek N, Nguyen P, Gibescu M., "Local market framework for exploiting flexibility from the end users", Int. Conf. Europe Energy Market, Jul. 2016, pp. 1-6.

[17] E. Amicarelli, T. Q. Tran, and S. Bacha, "Flexibility service market for active congestion management of distribution networks using flexible energy resources of microgrids,” 2017 IEEE PES Innovative Smart Grid Technologies Conf. Europe, Torino, 2017, pp. 1-6.

[18] A. Esmat, J. Usaola, and M. Á. Moreno, "Distribution-level flexibility market for congestion management”, Energies, vol. 11, no. 5, Apr. 2018.

[19] A. Esmat, P. Pinson, and J. Usaola, "Decision support program for congestion management using demand side flexibility," in Proc. IEEE Power Tech. Conf. Manchester, Manchester, U.K., 2017, pp. 1-6.

[20] A. Esmat, J. Usaola, and M. Á. Moreno, “A decentralized local flexibility market considering the uncertainty of demand”, Energies, vol. 11, no. 8, Aug. 2018.

[21] P. Olivella-Rosell, P. Lloret-Gallego, Í. Munné-Collado, et al., "Local flexibility market design for aggregators providing multiple flexibility services at distribution network level”, Energies, vol. 11, no. 44, Apr. 2018.

[22] S. Boyd, N. Parikh, E. Chu, B. Peleato, and J. Eckstein, "Distributed optimization and statistical learning via the alternating direction method of multipliers," Foundations and Trends in Machine Learning, vol. 3, no. 1, pp. 1-122, Jan. 2010.

[23] R. R. Nejad and W. Sun, "Distributed load restoration in unbalanced active distribution systems," IEEE Trans. Smart Grid, vol. 10, no. 5, pp. 5759-5769, Sep. 2019.

[24] Q. Peng and S. H. Low, "Distributed optimal power flow algorithm for radial network, I: Balanced single-phase case," IEEE Trans. Smart Grid, vol. 9, no. 1, pp. 111-121, Jan. 2018.

[25] Y. Wang, L. Wu, and S. Wang, "A fully-decentralized consensus-based ADMM approach for DC-OPF with demand response," IEEE Trans. Smart Grid, vol.8, no. 6, pp. 2637-2647, Nov. 2017.

[26] S. Huang, Q. Wu, Y. Gao, X. Chen, B. Zhou, and C. Li, "Distributed voltage control based on ADMM for large-scale wind farm cluster connected to VSC-HVDC," IEEE Trans. Sustain. Energy, vol. 11, no.2, pp. 584-594, Apr. 2020.

[27] W. Zheng, W. Wu, B. Zhang, and C. Lin, "Distributed optimal residential demand response considering operational constraints of unbalanced distribution networks", IET Gener. Transm. Distrib., vol. 12, no. 9, pp. 1970-1979, May 2018.

[28] P. Olivella-Rosell, E. Bullich-Massagué, , M. Aragüés-Peñalba, A. Sumper, S. Ø. Ottesen, J. A. Vidal-Clos, and R. Villafáfila-Robles, "Optimization problem for meeting distribution system operator requests in local flexibility markets with distributed energy resources", Applied Energy, vol. 210, pp. 881-895, Jan. 2018.

[29] S. Huang, Q. Wu, Z. Liu, and A. H. Nielsen, "Review of congestion management methods for distribution networks with high penetration of distributed energy resources,” in Proc. 2014 IEEE PES Innovative Smart Grid Technologies, Europe, pp. 1-6.

[30] U.S. Department of Energy, "Global plug-in light vehicle sales increased by about 80\% in 2015," 2016. Available: https://www.energy.gov/eere/vehicles/fact-918-march-28-2016-global-plug-light-vehicle-sales-increased-about-80-2015

[31] European heat pump association, "The European heat pump market has achieved double-digit growth for the fourth year in a row," 2019. Available: https://www.ehpa.org/market-data/

[32] T. Wolf, "Model-based assessment of heat pump flexibility," Master thesis, UPPSALA University, Apr. 2016. 
[33] R. Deng, X. Yue, D. Huo, Y. Liu, Y. Huang, C. Huang, and J. Liu, "Exploring flexibility of electrical vehicle aggregators as energy reserve,” vol. 184, pp. 106305, Electric Power Systems Research, Jul. 2020.

[34] Bolognani S and Zampieri S, "On the existence and linear approximation of the power flow solution in power distribution networks," IEEE Trans. Power Syst. vol. 31, pp. 163-172, Jan. 2016.

[35] M. Doostizadeh, F. Aminifar, H. Lesani, and H. Ghasemi, "Multi-area market clearing in wind-integrated interconnected power systems: A fast parallel decentralized method”, Energy Conversion and Management, vol. 113, pp. 131-142, Apr. 2016.

[36] R. N. Allan, R. Billinton, I. Sjarief, L. Goel, and K. S. So, “A reliability test system for educational purposes-basic distribution system data and results," IEEE Trans. Power Syst., vol. 6, no. 2, pp. 813-820, May 1991. 